USC(NT)-93-6

Submitted to Internat'l J. Mod. Phys. E

\title{
Neutrino-Nucleus Reactions
}

\author{
Kuniharu Kubodera用 \\ Department of Physics and Astronomy \\ University of South Carolina \\ Columbia, SC 29208, U.S.A. \\ and \\ Satoshi Nozawa \\ Department of Physics \\ Queen's University \\ Kingston, Canada, K4L 3N6
}

\begin{abstract}
The current status is reviewed of theoretical treatments of neutrino-nucleus reactions that are relevant to the detection of astrophysical neutrinos. Various nuclear physics aspects involved in the evaluation of the neutrino-nucleus reaction cross sections are critically surveyed.
\end{abstract}

\footnotetext{
*Work supported in part by National Science Foundation Grant No. PHYS-9006844.
} 


\section{Chapter 1 Introduction}

Although many topics can come under the title: "Neutrino-Nucleus Reactions", we shall be concerned here with neutrino-nucleus reactions that pertain to the terrestrial observation of astrophysical neutrinos. The neutrinos play fundamental roles in various astrophysical phenomena. The wealth of these phenomena is eruditely described in, e.g., Bahcall [1] and Fukugita [2]. Obviously, terrestrial experiments to detect these neutrinos are highly valuable sources of astrophysical information, and observational neutrino astrophysics is an extremely rapidly expanding research field [3]. It is useful to distinguish three different energy regimes of the astrophysical neutrinos. (i) The low energy region $\left(E_{\nu} \lesssim 20 \mathrm{MeV}\right)$, which includes the solar neutrinos and the lower energy part of the supernova neutrinos. (ii) The medium-energy region $\left(20 \mathrm{MeV} \lesssim \mathrm{E}_{\nu} \lesssim 50 \mathrm{MeV}\right)$ exemplified by the higher energy region of supernova neutrinos. (iii) The high-energy region $\left(50 \mathrm{MeV} \lesssim \mathrm{E}_{\nu}\right)$ represented by the solar flare neutrinos and atmospheric neutrinos, whose energy can reach as high as $1 \sim 2$ $\mathrm{GeV}$. Neutrino-nucleus reactions offer a great variety of ways to detect these astrophysical neutrinos. Generally, the following types of neutrino-nucleus reactions are possible:

$$
\begin{aligned}
& \nu_{\ell}+{ }^{Z} A \rightarrow \ell^{-}+{ }^{Z+1} A, \\
& \overline{\nu_{\ell}}+{ }^{Z} A \rightarrow \ell^{+}+{ }^{Z-1} A, \\
& \nu_{\ell}+{ }^{Z} A \rightarrow \nu_{\ell}+{ }^{Z} A, \\
& \overline{\nu_{\ell}}+{ }^{Z} A \rightarrow \overline{\nu_{\ell}}+{ }^{Z} A .
\end{aligned}
$$

In the charged-current $(\mathrm{CC})$ reactions, eqs. (11),(2), the script $\ell$ is limited to the electron family unless the incident energy is high enough to produce a muon or a tau-lepton, whereas the neutral-current (NC) reactions, eqs.(3),(仿), can always occur for $\ell=e, \mu$ and $\tau$. The final nuclear state $A$ can in general be either bound or unbound (if the latter is allowed energetically), and all these will contribute to inclusive events in which only the final leptons are monitored. On the other hand, in a particular type of experiment such as a radiochemical experiment, only the contribution of particle-bound final states will be registered. 
To set the stage, we first give a brief survey of the solar neutrino problem. The Sun generates its energy primarily by changing protons into $\alpha$-particles. The actual reaction chain through which $p+p+p+p \rightarrow{ }^{4}$ He takes place involves many intermediate nuclear reactions, some of which are weak-interaction processes emitting neutrinos. The standard solar model (SSM) [1, 国] gives a definite prediction of the neutrino flux coming from each of these neutrino-emitting reactions. The first observation of the solar neutrinos was achieved by Davis and his collaborators [5] through a radiochemical counting of ${ }^{37} \mathrm{Ar}$ produced in the reaction $\nu_{e}+{ }^{37} \mathrm{Cl} \rightarrow e^{-}+{ }^{37} \mathrm{Ar}$. According to the SSM, the ${ }^{37} \mathrm{Cl}$ detector of Davis et al's Homestake facility should register $7.9 \pm 2.6 \mathrm{SNU}\left(1 \mathrm{SNU}=10^{-37}\right.$ events per target atom per second), but the observed value is only $2.1 \pm 0.9 \mathrm{SNU}$ [5]-[9]. This clear deficit of the neutrino flux is the solar neutrino problem, which is one of the most fundamental issues in today's astrophysics. An independent confirmation of the deficit of the solar neutrino flux has been obtained by the Kamiokande experiment [10, 11, 12], in which the neutrino flux is monitored through the leptonic reaction $\nu_{e}+e^{-} \rightarrow \nu_{e}+e^{-}$occurring in the large water Cerenkov counter. The neutrino flux observed by the Kamiokande [12] is $0.46 \pm 0.05$ (stat) \pm 0.06 (syst) times the SSM prediction, corroborating the existence of the solar neutrino problem. One thing to be noted here is that the neutrino detection thresholds of the Homestake and Kamiokande facilities are rather high. The Kamiokande with $E_{\text {thresh }}=7.5 \mathrm{MeV}$ is sensitive only to the neutrinos coming from the reaction ${ }^{8} \mathrm{~B} \rightarrow{ }^{8} \mathrm{Be}+e^{+}+\nu_{e}\left(E_{\nu}^{\max }=15 \mathrm{MeV}\right)$, whereas the chlorine detector is sensitive to the ${ }^{8} \mathrm{~B}$ neutrinos and, to a much lesser degree, to the neutrinos coming from ${ }^{7} \mathrm{Be}+e^{-} \rightarrow{ }^{7} \mathrm{~B}+\nu_{e}$. Since ${ }^{8} \mathrm{~B}$ is produced only towards the end of the solar thermonuclear reaction chain, the SSM prediction of the neutrino flux due to ${ }^{8} \mathrm{~B}$ is extremely sensitive to the fine details of solar models. By contrast, the neutrino flux due to the primary solar thermonuclear reaction $p+p \rightarrow{ }^{2} \mathrm{H}+e^{+}+\nu_{e}$ reflects more directly the basic features of the SSM. However, since the maximum neutrino energy for this process is small $\left(E_{\nu}^{\max }=0.420 \mathrm{MeV}\right)$, the detection of the pp-neutrinos requires a neutrino absorption reaction with a very low Q-value. The best target so far 
proposed for this purpose is ${ }^{71} \mathrm{Ga}$. The threshold of the $\nu_{e}+{ }^{71} \mathrm{Ga} \rightarrow e^{-}+{ }^{71} \mathrm{Ge}$ reaction is $E_{\mathrm{th}}=0.2332 \mathrm{MeV}$, and the $\mathrm{SMM}$ prediction for the ${ }^{71} \mathrm{Ga}$ target is $132_{-17}^{+20} \mathrm{SNU}$ [4], of which more than half is due to the pp neutrinos. Two groups have recently published the results of Ga experiments. The GALLEX collaboration [13] reports $[83 \pm 19(1 \sigma) \pm 8$ (syst) SNU], and the SAGE collaboration [14, 15] gives [ $58_{-24}^{+17} \pm 14$ (syst) SNU], both significantly below the SSM value. A wide range of possible solutions to the solar neutrino problem have been proposed so far. These "solutions" fall into two categories. In the first category (solar-model explanations) a possible deviation from the SSM prediction on the interior properties of the Sun is sought. In the second category (particle-physics explanations), the observed neutrino flux deficiency is ascribed to unusual properties of the neutrinos.

Bahcall and Bethe [16] have recently carried out a systematic study of solar-model explanations. They studied a collection of 1000 precise solar models in which each input parameter (the principal nuclear reaction rates, the solar composition, the solar age, and the radiative opacity) for each model was drawn randomly from a normal distribution with the mean value and standard deviation appropriate to that variable. None of these 1000 solar models was compatible with the results of the chlorine or the Kamiokande experiments. Even if the solar models were artificially modified to reproduce a ${ }^{8} \mathrm{~B}$ neutrino flux in agreement with the Kamiokande experiment, none of these fudged models agreed with the chlorine observation. Furthermore, the GALLEX and SAGE experiments were found to disagree with the prediction of the solar models by $2 \sigma$ and $3 \sigma$, respectively. Thus, the totality of the existing solar neutrino data seems to strongly indicate that the solar neutrino problem has at least part of its origin in the hitherto unknown properties of the neutrinos.

The particle-physics explanations invoke, in one form or another, a mechanism to convert electron neutrinos into other particles (neutrino oscillation) so that not all of the original $\nu_{e}$ 's produced in the core of the Sun would reach the terrestrial detectors (for a review, see e.g. [17]). The most promising particle-physics solution so far proposed is the Mikheyev-Smirnov-Wolfenstein (MSW) effect [18, 19], which can be summarized as follows. 
The three neutrino states, $\nu_{e}, \nu_{\mu}$ and $\nu_{\tau}$, are assumed to have different masses, with the electron neutrino the lightest. In the solar core with high electron densities, the neutrinos acquire effective masses through the neutrino-electron interactions. The neutral-current interaction simply gives a universal shift of the neutrino masses, but the charged-current interaction, which in the absence of muons and $\tau$-leptons is operative only for $\nu_{e}$, increases the $m_{\nu_{e}}$ selectively so that, for sufficiently high electron densities, the effective electron neutrino mass can become heavier than those of the other neutrinos. If the Hamiltonian involves a piece that causes lepton-flavor mixing, then in a critical region where $m_{\nu_{e}}^{\text {eff }} \sim m_{\nu_{\mu}}^{\text {eff }}$, or $m_{\nu_{e}}^{\mathrm{eff}} \sim m_{\nu_{\tau}}^{\mathrm{eff}}$, it is possible for $\nu_{e}$ to transform into the other neutrino species via resonant transitions. This phenomenon is called the MSW effect 18, 19]. The parameters characterizing the MSW mechanism are the neutrino mass differences in free space and the strengths of flavor mixings. By adjusting these parameters one can vary the region of the electron neutrino spectrum that is changed into the other neutrinos through the resonant transitions. The contemporary literature abounds in articles that deal with the determination of the parameters characterizing the MSW model or its variants. At present, the bare neutrino mass difference of about $10^{-2}$ to $10^{-3} \mathrm{eV}$ with appropriate corresponding mixing strengths is compatible with the existing data. However, this does not constitute unambiguous evidence that the MSW mechanism is operative in the Sun; the direct confirmation of the MSW effect needs an additional set of experiments. Neutrino-nucleus reactions are of great importance in this context, since in some favorable cases they allow us to identify various types of neutrino reactions individually. Of particular significance is the detection of neutral-current (NC) reactions, eq.(3)); since the $\mathrm{NC}$ reaction rate is the same regardless of neutrino species, a measurement of the NC reactions gives the bolometric flux of all neutrino species [20, 21, 22]. Therefore, by combining information on the NC reactions with that on the CC reactions, one can obtain definitive information on neutrino oscillation, or more specifically, on the MSW effect. In the planned Sudbury Neutrino Observatory (SNO) [23, 24], which uses a 1,000-tonne, heavy-water Cerenkov counter, one aims at the separate 
registration of the $\mathrm{CC}$ and $\mathrm{NC}$ reactions on the deuteron with the detection threshold as low as $E_{\nu}=5 \mathrm{MeV}$. The data obtained at the $\mathrm{SNO}$ will provide unambiguous information on the neutrino flux for individual flavors and consequently on the neutrino oscillation. There are other nuclear targets which are important in this context, and we will discuss them later in the text.

The neutrino-nucleus reactions are not the only place where neutral-current interactions appear. The leptonic reactions:

$$
\begin{aligned}
& \nu_{\ell}+e^{-} \rightarrow \nu_{\ell}+e^{-}, \\
& \bar{\nu}_{\ell}+e^{-} \rightarrow \bar{\nu}_{\ell}+e^{-},
\end{aligned}
$$

where $\ell=e, \mu$ or $\tau$, do involve the neutral-current contributions. More specifically, for $\ell=e$, the reactions can occur through both the charged and neutral currents, whereas for $\ell=\mu$ and $\tau$ only the neutral current will participate. Therefore, water Cerenkov counters and liquid scintillation counters in which these leptonic reactions provide main signals do count both neutral-current (NC) and charge-current (CC) events. It is to be noted, however, that the $\mathrm{NC}$ and $\mathrm{CC}$ events cannot be registered separately by monitoring the leptonic reactions, eqs.(5),(6). This situation makes the use of nuclear targets particularly attractive.

We now turn to astrophysical neutrinos other than the solar neutrinos. The large water Cerenkov counters at the Kamiokande and IMB allowed the first observation of the supernova neutrinos coming from SN1987A. For this spectacular success it was crucial that these facilities had sufficiently high time resolutions to clearly define supernova-related neutrino events. Since all the existing nuclear-target detectors use the radio-chemical counting method, their time resolution is not sufficient to allow the monitoring of supernova neutrinos. In the near future, however, counter experiments involving nuclear targets will become possible. The above-mentioned Sudbury Neutrino Observatory (SNO) [23, 24] is a notable example. As will be discussed in more detail later, the SNO's ability to detect the NC reactions with high time-resolution can be important in the context of the supernova neu- 
trinos as well. We will also discuss some other nuclear targets which can be very useful in connection with supernova neutrino detection.

In considering the high-energy astrophysical neutrinos group (iii) in the abovedescribed classification], it is important to realize that, as the incident neutrino energy increases, the nuclear reactions become progressively more important as compared to the leptonic processes. This implies that even the water Cerenkov counter and liquid scintillation counter, which for low-energy neutrinos primarily detect the electrons from the leptonic reactions, will start detecting the nuclear reactions [25]. As will be discussed in the following, this offers yet another interesting use of these facilities. A notable example is the water Cerenkov counter measurement of the ratio of the muonic neutrino flux to the electronic neutrino flux in the atmospheric neutrinos.

One of the fundamental prerequisites for nuclear targets envisaged as neutrino detectors is that reliable estimates of the relevant reaction cross sections be available. This presents an interesting and challenging task to nuclear physicists. The great variety of relevant nuclear targets and the wide energy range of the astrophysical neutrinos to be considered necessitate the use of many different approaches that can be found in the nuclear physics arsenal. In this review, we survey the current status of theoretical attempts at obtaining reliable estimates of the neutrino-nucleus reactions of astrophysical interest. To highlight the basic aspects of various approaches so far used and their possible limitations, we shall organize our topics according to the calculational methods involved. We describe in chapter 2 the direct microscopic calculation which is relevant to the lightest nuclei. In chapter 3 we discuss a model-independent determination of the cross sections based on

\footnotetext{
${ }^{\dagger}$ The cross sections of the leptonic processes, eqs. (5),(6), by contrast are accurately known from the standard electroweak theory. There is in fact one particular type of neutrino-nucleus reaction for which the cross section can be determined mode-independently; for the coherent scattering $\nu+A($ gnd $) \rightarrow \nu+A$ (gnd), the cross section is determined by the number of nucleons in $A$. The coherent process plays an important role in dark matter search [26] as well as in certain types of solar neutrino experiments 27, 28, 29, but we will not discuss the coherent scattering here. For a review of this topic, see [1].
} 
the elementary-particle treatment. Chapter 4 deals with the empirical effective operator method. The use of $(\mathrm{p}, \mathrm{n})$ reactions as calibrators of the Gamow-Teller matrix elements is surveyed in chapter 5. Chapters 6 and 7 are concerned with high-energy neutrino-nucleus reactions. The Fermi gas model and its possible refinement are discussed in chapter 6 , while the treatment of semi-inclusive reaction is addressed in chapter 7. Additional remarks are made in chapter 8. 


\section{Chapter 2 "Ab initio" calculations based on realistic wave functions}

In the so-called realistic description of nuclei one works with the nuclear Hamiltonian

$$
H_{N}=\sum_{i=1}^{A} T_{i}+\sum_{i, j}^{A} V_{i j},
$$

where $A$ is the mass number, $T_{i}$ is the nucleon kinetic energy, and $V_{i j}$ is the realistic nucleonnucleon potential. Although $H_{N}$ is called the realistic Hamiltonian due to the fact that $V_{i j}$ reproduces the observed properties of the two-nucleon systems reasonably well, $H_{N}$ is an effective Hamiltonian of highly operational nature. To arrive at $H_{N}$ starting from the fundamental QCD picture is far from trivial. First, the dynamical degrees of freedom of quarks and gluons need to be translated into the effective degrees of freedom of hadrons. Second, the Hilbert space of hadrons must be truncated down to those of non-relativistic nucleons interacting via potentials. Despite these fundamental problems, it is generally believed that solutions for the Schrödinger equation with the Hamiltonian $H_{N}$ give reasonably reliable nuclear wave functions, and it is usually considered as an ab initio approach to calculate matrix elements of nuclear electromagnetic or weak-interaction processes with the use of these wave functions along with the standard non-relativistic reduction of the single-nucleon responses to the relevant currents [30, 31]. Two major problems encountered in this ab initio approach are as follows. First, it is in general extremely difficult to obtain an exact solution of the $A$-body Schrödinger equation $H_{N} \Psi(1,2, \ldots A)=E \Psi(1,2, \ldots A)$, and hence one is usually forced to work with rather drastically truncated model wave functions $\Psi_{0}$; shell-model wave functions are the most commonly used and most successful example. Now, if the matrix element of a nucleon observable $\mathcal{O}_{N}$ is calculated using model wave functions $\Psi_{0}^{i}$ and $\Psi_{0}^{f}$, one expects $<\Psi^{f}\left|\mathcal{O}_{N}\right| \Psi^{i}>\neq<\Psi_{0}^{f}\left|\mathcal{O}_{N}\right| \Psi_{0}^{i}>$. This difference is called corepolarization effect. Another point is that eliminating all hadronic degrees of freedom but those of the nucleons is an approximation and, even if one can calculate $<\Psi^{f}\left|\mathcal{O}_{N}\right| \Psi^{i}>$ exactly, it may still differ from the corresponding true matrix element. One usually accounts for this difference by adding to $\mathcal{O}_{N}$ many-body operators $\mathcal{O}^{\prime}$, which represent the 
effects of the eliminated hadronic degrees of freedom. These additional terms are called the exchange-current operators.

Now, to calculate the core-polarization and exchange-current effects in complex nuclei is one of the most challenging problems in nuclear physics and, although many important achievements have so far been made in this domain, the problem is far from solved. However, nuclei with small mass numbers $(A \leq 4)$ offer important exceptions. For these lightest nuclei one can obtain sufficiently "realistic" wave functions either by directly solving the Schrödinger equation or through variational calculations, eliminating thereby the core-polarization problem. For these systems, therefore, one can carry out ab initio calculations of the transition matrix elements of electromagnetic and weak-interaction processes, provided a reasonably reliable method to incorporate the exchange-current effects exists.

\section{1. $\quad \nu-d$ reactions}

The $\mathrm{A}=2$ systems are the simplest nuclei, for which one can obtain reasonably reliable wave functions corresponding to the realistic Hamiltonian eq.(7). This allows us to estimate with high accuracy the cross sections of the neutrino-deuteron reactions. These cross sections are important for deriving useful astrophysical information from the experimental data that will soon become available at the Sudbury Neutrino Observatory (SNO) [23, 24]. The neutrino-deuteron reactions that are relevant to the SNO are:

$$
\begin{gathered}
\nu+d \rightarrow \nu^{\prime}+n+p \\
\bar{\nu}+d \rightarrow \bar{\nu}+n+p \\
\nu_{e}+d \rightarrow e^{-}+p+p, \\
\bar{\nu}_{e}+d \rightarrow e^{+}+n+n,
\end{gathered}
$$

As mentioned earlier, one of the great advantages of the SNO is its capability to detect both the neutral- and charged-current reactions and record them separately, and this is crucially important [22] for proving that the MSW effect 18] is indeed a solution for the 
solar neutrino problem [1].

The earlier calculations [32, 33, 34] were mainly meant for low-energy solar neutrinos and therefore included only s-wave for the final N-N relative motion. Extended calculations that take account of higher partial waves and, correspondingly, the contributions of the forbidden-type transitions were carried out by Ying, Haxton and Henley [35] (to be referred to as YHH1) and by Tatara, Kohyama and Kubodera [36] (to be referred to as TKK) up to $E_{\nu}=170 \mathrm{MeV}$. Unfortunately, the cross sections obtained in these two detailed calculations show alarmingly large discrepancies for higher energies; the YHH1 results become significantly larger than those of TKK around $E_{\nu} \sim 20 \mathrm{MeV}$ and the cross section ratios reach $\sim 2$ towards $E_{\nu}=170 \mathrm{MeV}$. To clarify the origin(s) of these discrepancies, Doi and Kubodera 37] (to be referred to as DK1) carried out a systematic comparison of the formalisms used in YHH1 and TKK. The numerical results of the independent calculation in DK1 37] suggested possible numerical errors in YHH1, and this suggestion was confirmed by the authors of YHH1. The results of a revised estimation by Ying, Haxton and Henley [38] (to be referred to as YHH2) are in essential agreement with those of TKK and DK1.

Beyond the $\sim 20 \%$ level precision, however, there still exist discrepancies among the results of TKK, DK1 and YHH2. The cross sections in TKK begin to be systematically smaller than the corresponding numbers in DK1 and YHH2 as $E_{\nu}$ surpasses $\sim 100 \mathrm{MeV}$, and the differences for some channels reach $\sim 20 \%$ towards $E_{\nu}=170 \mathrm{MeV}$. It has been demonstrated in DK1 that this discrepancy arises because TKK dropped the term quadratic in $\boldsymbol{J}^{V}$, the space component of the vector current. At low energies, the contribution of $\boldsymbol{J}^{V}$ is known to be much less important than that of the time-component $\rho_{V}$. TKK, assuming that this behavior would persist for higher energies, ignored the quadratic term $\left|\boldsymbol{J}^{V}\right|^{2}$ that appears in squaring the transition matrix element to obtain the cross section. According to DK1, however, the contribution of $\boldsymbol{J}^{V}$ is comparable to or even larger than that of $\rho_{V}$ for $E_{\nu} \gtrsim 10 \mathrm{MeV}$, and ignoring $\left|\boldsymbol{J}^{V}\right|^{2}$ leads to an underestimation of the cross sections by up to $\sim 20 \%$. Meanwhile, $\sigma\left(\bar{\nu}_{e}+d \rightarrow e^{+}+n+n\right)$ of DK1 becomes progressively larger 
than the corresponding results in TKK as $E_{\nu}$ decreases from $7 \mathrm{MeV}$; e.g., at $E_{\nu}=6 \mathrm{MeV}$, $\sigma\left(\bar{\nu}_{e}+d \rightarrow e^{+}+n+n\right)_{\mathrm{DK} 1} / \sigma\left(\bar{\nu}_{e}+d \rightarrow e^{+}+n+n\right)_{\mathrm{TKK}}=1.07$. (At this low energy the contribution of $\left|\boldsymbol{J}^{V}\right|^{2}$ ignored in TKK is completely negligible.) A recent study by Doi and Kubodera [39] (to be referred to as DK2) indicates that this behavior is caused by the approximate Q-value expression used for this channel in DK1. As far as comparison with YHH2 is concerned, we remark that the exchange-currents effects are altogether ignored in YHH2. This is estimated [36] to lead to a general underestimation of the cross sections by up to $\sim 5 \%$. Thus each of the published works, TKK [36], DK1 [37] and YHH2 [38], has some point(s) to be improved upon.

Kohyama and Kubodera [40] have extended the calculation of Tatara et al. [36] by including the contributions of the $\left|J_{V}\right|^{2}$ terms. The results of Kohyama and Kubodera 40] and those of DK2 [39] have been found to agree with each other within $2 \%$. Since these two calculations are independent, both in the calculational methods and in choosing various input parameters, it is reassuring that their results agree to this degree. (According to TKK, different choices of the nuclear potential from among realistic nucleon-nucleon interactions affects the cross sections by up to $1 \%$.) We tabulate in table 1 in Appendix the cross sections for the reactions eqs.(8)-(11) obtained by Kohyama and Kubodera 40]; $5 \%$ uncertainties are attached to the cross sections. Table 1 represents the best available estimates of the neutrino-deuterium cross sections up to $E_{\nu}=170 \mathrm{MeV}$.

One generally expects that the ratios $R_{\nu} \equiv \sigma\left(\nu_{e}+d \rightarrow e^{-}+p+p\right) / \sigma\left(\nu+d \rightarrow \nu^{\prime}+n+p\right)$ and $R_{\bar{\nu}} \equiv \sigma\left(\bar{\nu}_{e}+d \rightarrow e^{+}+n+n\right) / \sigma(\bar{\nu}+d \rightarrow \bar{\nu}+n+p)$ are much less sensitive to the nuclear models than the absolute cross sections themselves. Indeed, the $R_{\nu}$ and $R_{\bar{\nu}}$ obtained by Kohyama and Kubodera 40] agree with the corresponding values given in DK2 [39] within $0.2 \%$. Therefore, it seems reasonably safe to assign $1 \%$ uncertainties to the calculated values of $R_{\nu}$ and $R_{\bar{\nu}}$.

The average cross section for the electronic neutrinos from the $\mu^{+}$decay calculated using the results of 40 is: $\left\langle\sigma\left(\nu_{e}+d \rightarrow e^{-}+p+p\right)>_{\mu^{+}-\text {decay }}=5.4 \times 10^{-41} \mathrm{~cm}^{2}\right.$. This is 
consistent with the observed value $\sigma=(5.2 \pm 1.8) \times 10^{-41} \mathrm{~cm}^{2}$ 41.

Apart from testing the MSW effect for the solar neutrinos, Bahcall et al. [34] considered the merit of the SNO in determining the masses of heavy-flavor neutrinos through arrival-time-delay measurements of supernova neutrinos. Some other possible uses of the SNO for solar and supernova neutrinos were discussed by Balantekin and Loreti [42]. The SNO can also be used to study neutrinos of extremely high energies such as solar-flare and atmospheric neutrinos. The above described "ab initio" calculation probably can be extended up to $E_{\nu} \sim$ several hundred $\mathrm{MeV}$, but its reliability will diminish rather significantly for these high energies. At yet higher energies, the results are expected to approach those of the high-energy approximation to be discussed in chapter 6 .

\subsection{Neutrino-production reaction on the lightest nuclei}

Although our review is primarily concerned with neutrino-nucleus reactions, this is a good place to discuss the latest development in the theoretical treatment of neutrinoproduction reactions on the lightest nuclei. As mentioned earlier, the pp reaction

$$
p+p \rightarrow d+e^{+}+\nu_{e}
$$

is the primary solar thermonuclear reaction and produces a dominant part of the solar neutrino flux [1]. The "hep" reaction

$$
{ }^{3} \mathrm{He}+\mathrm{p} \rightarrow{ }^{4} \mathrm{He}+\mathrm{e}^{+}+\nu_{\mathrm{e}}
$$

contributes only a tiny portion of the solar neutrino flux [1], but the maximum neutrino energy of the hep process $\left(E_{\nu}^{\max }=18.77 \mathrm{MeV}\right)$ is the largest of all the solar neutrinos. Carlson et al. 43 have carried out a detailed ab initio calculation of the cross sections for these reactions, using as a nucleon-nucleon interaction the Argonne $v_{14}$ potential. To reduce the uncertainty in the axial exchange current operator, its matrix element has been adjusted so as to reproduce the measured Gamow-Teller matrix element for tritium $\beta$-decay. For the pp reaction, the calculated astrophysical $S$ factor and its derivative at zero energy 
are: $S(E=0)=4.00 \times 10^{-25} \mathrm{MeV}$ b, and $d S(E) /\left.d E\right|_{E=0}=4.07(1 \pm 0.051) \times 10^{-25} \mathrm{MeV}$ $\mathrm{b}$; these are close to the values quoted in 断. Carlson et al. find that exchange-current contribution enhances the capture rate by $1.5 \%$. This is considerably smaller than the enhancement found in earlier works [44, 45]. In the absence of Coulomb distortion the transition matrix element of the pp reaction is just the hermitian conjugate of that of the $\nu_{e}+d \rightarrow e^{-}+p+p$ reaction. For the thermal pp reaction, however, the Coulomb repulsion between the two protons is extremely important and responsible for the reduction of exchange-current contribution as compared with the typical values found for $\nu_{e}+d \rightarrow$ $e^{-}+p+p$ reaction 36$]$.

As for the ${ }^{3} \mathrm{He}\left(p, e^{+} \nu\right)^{4} \mathrm{He}$ reaction rate, Carlson et al. 43 obtained $S(E=0)=$ $1.3 \times 10^{-23} \mathrm{MeV}$ b, which is smaller by a factor of $\sim 6$ than the values obtained earlier by Werntz and Brennan [46] and by Tegner and Bargholtz [47]. This change is caused primarily by the exchange current effect, which cancels the impulse approximation contribution. This is one of the most dramatic examples of the exchange-current effect. 


\section{Chapter $3 \quad$ Elementary-particle treatment (EPT)}

The elementary-particle treatment (EPT) was first introduced by Kim and Primakoff 48] and by Fujii and Yamaguchi [49]. In EPT, instead of describing nuclei in terms of nucleons or other constituents, one treats nuclei as "elementary" particles with given quantum numbers. A transition matrix element for a given process is parametrized in terms of the nuclear form factors solely based on the transformation properties of the relevant current and nuclear states [48, 50, 51]. Insofar as all of these nuclear form factors can be deduced empirically from experimental data, one can make a totally model-independent prediction on every observable for the given transition. In fact, this requirement is an extremely stringent one, and so far the EPT calculation has been carried out completely only for the triad of ${ }^{12} \mathrm{~B}-{ }^{12} \mathrm{C}-{ }^{12} \mathrm{~N}$.

This particular $A=12$ case, however, deserves special attention. One reason is that the ${ }^{12} \mathrm{C}$ nucleus, which is abundantly contained in ordinary liquid scintillators, can be a very important nuclear target in neutrino astrophysics. Although the threshold energies of the neutrino reactions on ${ }^{12} \mathrm{C}$ are too high for solar neutrino detection, they are low enough for observing energetic neutrinos originating from stellar collapses. The neutrino energies up to $E_{\nu} \sim 100 \mathrm{MeV}$ are expected to be of relevance for stellar collapse neutrinos. Another reason is provided by the intensive programs of beam-dump neutrino experiments at Los Alamos [52, 53, 54, 55] and the Rutherford Laboratory [76, 57]. Some of these experiments directly aim at observing the neutrino $-{ }^{12} \mathrm{C}$ reactions. In other particle-physics experiments 52 main interest is in the $\nu-e$ scattering but here also, to eliminate the dominant background due to the neutrino $-{ }^{12} \mathrm{C}$ reaction, one needs to know its cross sections with reasonable accuracy.

The processes of relevance here are the superallowed neutral-current (NC) reactions

$$
\begin{aligned}
& \nu+{ }^{12} \mathrm{C}\left(0^{+} ; \text {gnd }\right) \rightarrow \nu^{\prime}+{ }^{12} \mathrm{C}^{*}\left(1^{+} ; 15.1 \mathrm{MeV}\right), \\
& \bar{\nu}+{ }^{12} \mathrm{C}\left(0^{+} ; \text {gnd }\right) \rightarrow \bar{\nu}^{\prime}+{ }^{12} \mathrm{C}^{*}\left(1^{+} ; 15.1 \mathrm{MeV}\right),
\end{aligned}
$$


and the superallowed charged-current $(\mathrm{CC})$ reactions

$$
\begin{aligned}
& \nu+{ }^{12} \mathrm{C}\left(0^{+} ; \text {gnd }\right) \rightarrow e^{-}+{ }^{12} \mathrm{~N}\left(1^{+} ; \text {gnd }\right), \\
& \bar{\nu}+{ }^{12} \mathrm{C}\left(0^{+} ; \text {gnd }\right) \rightarrow e^{+}+{ }^{12} \mathrm{~B}\left(1^{+} ; \text {gnd }\right) .
\end{aligned}
$$

The final nuclear states of the above reactions form a triad of $J^{P}=1^{+}, T=1$ states. The existence of these superallowed transitions is favorable for simultaneous monitoring of the NC- and CC-reactions. (The elastic scattering leading to ${ }^{12} \mathrm{C}\left(0^{+}\right.$; gnd) is unobservable in normal circumstances.) Fukugita, Kohyama and Kubodera [58] showed that EPT allows the completely model-independent determination of the cross sections for the reactions eqs.(14)(17) for $E_{\nu} \lesssim 100 \mathrm{MeV}$. A similar treatment was presented by Mintz and Pourkaviani [59, 60]. Although the use of EPT is at present limited to the transitions listed above, this is not an obstacle for monitoring these exclusive processes, as discussed in [58]. Here we summarize the calculation of Fukugita et al. [58].

The effective Hamiltonian responsible for the $\mathrm{NC}$ reactions is given by

$$
H_{e f f}=G_{F} / \sqrt{2} J_{\mu}^{3} L_{\mu}^{3}
$$

where $G_{F}$ is the Fermi constant, $L_{\mu}^{3}=\bar{\psi}_{\nu} \gamma_{\mu}\left(1-\gamma_{5}\right) \psi_{\nu}$, and $J_{\mu}^{3}=\left(1-2 \sin ^{2} \theta_{W}\right) V_{\mu}^{3}+A_{\mu}^{3}$, with $\theta_{W}$ being the Weinberg angle. (The isoscalar neutral current, which cannot cause nuclear excitations, has been dropped.) The effective Hamiltonian for the CC reactions is given by

$$
H_{e f f}=\left[G_{F} \cos \theta_{c} / \sqrt{2}\right] J_{\mu}^{( \pm)} L_{\mu}^{(\mp)}
$$

where $\theta_{c}$ is the Cabibbo angle; $L_{\mu}^{(-)}=\bar{\psi}_{e} \gamma_{\mu}\left(1-\gamma_{5}\right) \psi_{\nu}$, and $L_{\mu}^{(+)}=L_{\mu}^{(-) \dagger} ; J_{\mu}^{( \pm)}=V_{\mu}^{( \pm)}+A_{\mu}^{( \pm)}$. The most general form of the matrix elements of the vector and axial-vector currents for the $\mathrm{NC}$ reactions is

$$
\begin{gathered}
<{ }^{12} \mathrm{C}\left(1^{+}\right) ; p_{b}\left|V_{\mu}^{3}(0)\right|{ }^{12} \mathrm{C}\left(0^{+}\right) ; p_{b}>=\frac{1}{2} \frac{\epsilon_{\mu \nu \lambda \sigma} q_{\nu} Q_{\lambda} \xi_{\sigma} F_{M}\left(q^{2}\right)}{2 M} \\
<{ }^{12} \mathrm{C}\left(1^{+}\right) ; p_{b}\left|A_{\mu}^{3}(0)\right|^{12} \mathrm{C}\left(0^{+}\right) ; p_{b}>=\frac{1}{2}\left[\xi_{\mu} F_{A}\left(q^{2}\right)+q_{\mu}(\xi \cdot q) \frac{F_{P}\left(q^{2}\right)}{m_{\pi}^{2}}+Q_{\mu}(\xi \cdot q) \frac{F_{T}\left(q^{2}\right)}{2 M}\right],
\end{gathered}
$$


where $q=p_{b}-p_{a}, Q=p_{b}+p_{a}, \xi$ is the polarization vector of the spin-1 nucleus, and $M=\left(M_{a}+M_{b}\right) / 2$ is the average of the initial and final nuclear masses. All information on nuclear dynamics is contained in the nuclear form factors, $F_{M}\left(q^{2}\right), F_{A}\left(q^{2}\right), F_{P}\left(q^{2}\right)$, and $F_{T}\left(q^{2}\right)$. Here the nuclear form factors are classified in the "cartesian" representation. An alternative formalism is described in [50, 62]. For the $\mathrm{CC}$ reactions the Wigner-Eckart theorem in isospin space leads to

$$
\begin{gathered}
<{ }^{12} \mathrm{~N}\left(1^{+}\right) ; p_{b}\left|V_{\mu}^{(+)}(0)\right|{ }^{12} \mathrm{C}\left(0^{+}\right) ; p_{b}>=\frac{\epsilon_{\mu \nu \lambda \sigma} q_{\nu} Q_{\lambda} \xi_{\sigma} F_{M}\left(q^{2}\right)}{2 M} \\
<{ }^{12} \mathrm{~N}\left(1^{+}\right) ; p_{b}\left|A_{\mu}^{(+)}(0)\right|{ }^{12} \mathrm{C}\left(0^{+}\right) ; p_{b}>=\xi_{\mu} F_{A}\left(q^{2}\right)+q_{\mu}(\xi \cdot q) \frac{F_{P}\left(q^{2}\right)}{m_{\pi}^{2}}+Q_{\mu}(\xi \cdot q) \frac{F_{T}\left(q^{2}\right)}{2 M} .
\end{gathered}
$$

Now, the contribution of the $F_{P}$ term to the observables is proportional to (lepton mass) ${ }^{2}$ and therefore can be dropped in the present energy regime $\left(E_{\nu} \lesssim 100 \mathrm{MeV}\right)$ for which muonproduction can be ignored. For the remaining nuclear form factors one can first consider their values at $q^{2}=0$, and examine their $q^{2}$-dependence later. Define $F_{M} \equiv F_{M}\left(q^{2}=0\right)$, $F_{A} \equiv F_{A}\left(q^{2}=0\right)$, and $F_{T} \equiv F_{T}\left(q^{2}=0\right) . \quad F_{M}$ can be determined from the width of the $\gamma$-decay ${ }^{12} \mathrm{C}^{*}\left(1^{+} ; 15.1 \mathrm{MeV}\right) \rightarrow{ }^{12} \mathrm{C}\left(0^{+}\right.$; gnd $)$, and the CVC. From $\Gamma_{\gamma}^{e x p}=38.5 \pm 0.8 \mathrm{MeV}$ 63], we obtain $F_{M}=(1.516 \pm 0.016) \times 10^{-3} \mathrm{MeV}^{-1} . F_{A}$ and $F_{T}$ can be determined from the $\beta^{+}$decay ${ }^{12} \mathrm{~N}\left(1^{+}\right.$; gnd $) \rightarrow{ }^{12} \mathrm{C}\left(0^{+}\right.$; gnd $)$and the $\beta^{-}$decay ${ }^{12} \mathrm{~B}\left(1^{+}\right.$; gnd $) \rightarrow{ }^{12} \mathrm{C}\left(0^{+}\right.$; gnd $)$. The $f t$-value of the $\beta$-decay is written as

$$
f t=\frac{2 \pi^{2} \ln 2}{m_{e}^{5}\left(G_{F} \cos \theta_{c}\right)^{2} F_{A}^{2}\left(1+\frac{2}{3} E_{0} F_{T} / F_{A}\right)},
$$

with $E_{0}$ the maximum $\beta$-particle energy. The measurement of the $\beta$-ray angular distribution from aligned ${ }^{12} \mathrm{~B}$ and ${ }^{12} \mathrm{~N}$ parent nuclei gives the angular correlation quantities $\alpha_{ \pm}$, which are related to $F_{T}$ as $[64,65,66]$.

$$
\alpha_{+}+\alpha_{-}=-\frac{4}{3} F_{T} / F_{A}
$$

The experimental values 67, 68], $\alpha_{-}=(0.02 \pm 0.12) \times 10^{-3} \mathrm{MeV}^{-1}$ and $\alpha_{+}=-(2.72 \pm$ $0.27) \times 10^{-3} \mathrm{MeV}^{-1}$, give $F_{T} / F_{A}=(2.01 \pm 0.23) \times 10^{-3} \mathrm{MeV}^{-1}$. Then the contribution 
of the $F_{T}$ term in eq. 24) is $(2 / 3) E_{0}\left(F_{T} / F_{A}\right)=0.02$ for $E_{0}=15 \mathrm{MeV}$. The importance of this term relative to 1 being smaller than typical isospin-symmetry breaking effects within nuclei $(\alpha Z \sim 6 \%)$, the $F_{T}$ term in eq. 24) can be ignored. Experimentally 63, $\log (f t)=4.067 \pm 0.002$ for ${ }^{12} \mathrm{~B} \rightarrow{ }^{12} \mathrm{C}$, and $\log (f t)=4.120 \pm 0.003$ for ${ }^{12} \mathrm{~N} \rightarrow{ }^{12} \mathrm{C}$. The difference between $(f t)^{+}$and $(f t)^{-}$gives a measure of the isospin symmetry breaking quoted above (insofar as there is no second-class currents). Using $f t=12444 \pm 830 \mathrm{~s}$, which is the average of $(f t)^{+}$and $(f t)^{-}$with the error corresponding to their difference, we obtain $F_{A}=0.711 \pm 0.024$

As $E_{\nu}$ grows, one must consider the $q^{2}$-dependence of the nuclear form factors. The total cross section for the NC reactions including the $q^{2}$-dependence is given by

$$
\begin{aligned}
\sigma= & \sigma_{0} F_{A}^{2} E_{\nu^{\prime}} I \\
\sigma_{0}= & 3 G_{F}^{2} / 2 \pi=2.53 \times 10^{-44} \mathrm{~cm}^{2} /(\mathrm{MeV})^{2}, \\
I= & \frac{1}{2} \int_{-1}^{1} d z f\left(\boldsymbol{q}^{2}\right)\left[\left(1-\frac{z}{3}\right) \pm \frac{4}{3}\left(E_{\nu}+E_{\nu^{\prime}}\right)\left(1-2 \sin ^{2} \theta_{W} W\right)(1-z) \frac{F_{M}}{F_{A}}\right. \\
& +\frac{2}{3}\left[E_{\nu^{\prime}} E_{\nu}\left(1-z^{2}\right)+(1-z) \boldsymbol{q}^{2}\right]\left(1-2 \sin ^{2} \theta_{W}\right)^{2}\left(\frac{F_{M}}{F_{A}}\right)^{2} \\
& \left.-\frac{2}{3} \Delta M(1+z) \frac{F_{T}}{F_{A}}+\frac{1}{3} \boldsymbol{q}^{2}\left({\frac{F_{T}}{F_{A}}}^{2}\right)\right],
\end{aligned}
$$

where the upper (lower) sign refers to the neutrino (anti-neutrino) reaction; $z=\cos \theta$ with $\theta$ being the neutrino scattering angle; $E_{\nu^{\prime}}=E_{\nu}-\Delta M$ with $\Delta M=15.1 \mathrm{MeV}$; $f\left(\boldsymbol{q}^{2}\right)=\left[F_{A}\left(q^{2}\right) / F_{A}\right]^{2}$ with $q^{2}=(\Delta M)^{2}-\boldsymbol{q}^{2}$. The cross sections for the CC reactions are given by

$$
\sigma=2 \sigma_{0} \cos \theta_{c} p_{e} E_{e} F^{ \pm}\left(Z_{f} ; E_{e}\right) F_{A}^{2} I
$$

where the upper (lower) sign refers to the neutrino (anti-neutrino) reaction. Here $I$ is as defined previously except the replacements $E_{\nu^{\prime}} \rightarrow E_{e}$, and $\left(1-\sin ^{2} \theta_{W}\right) \rightarrow 1 ; F^{ \pm}\left(Z_{f} ; E_{e}\right)$ denotes the Fermi function for the Coulomb correction. $E_{e}=E_{\nu}-\Delta M$, with $\Delta M=16.827$ $\operatorname{MeV}(13.880 \mathrm{MeV})$ for ${ }^{12} \mathrm{C} \rightarrow{ }^{12} \mathrm{~N}\left({ }^{12} \mathrm{C} \rightarrow{ }^{12} \mathrm{~B}\right)$. The above expression has been obtained by assuming that the $q^{2}$-dependences of $F_{M}\left(q^{2}\right)$ and $F_{T}\left(q^{2}\right)$ are the same as that of $F_{A}\left(q^{2}\right)$. 
From an EPT analysis of the ${ }^{12} \mathrm{C}+\mu^{-} \rightarrow{ }^{12} \mathrm{~B}+\nu_{\mu}$ process, Nozawa et al. [69] showed that $F_{A}\left(q^{2}\right) / F_{A}=F_{M}\left(q^{2}\right) / F_{M}$ holds within a $10 \%$ accuracy at least up to $q^{2}=0.74 m_{\mu}^{2}$. For $F_{T}\left(q^{2}\right)$ no direct experimental information is available but, since its contribution is minor, its precise form has little significance. Now, $f\left(\boldsymbol{q}^{2}\right) \cong\left[F_{M}\left(q^{2}\right) / F_{M}\right]^{2}$ can be determined model-independently from $F_{M}\left(q^{2}\right)$ obtained from inelastic electron scattering: $e+{ }^{12} \mathrm{C} \rightarrow$ $e^{\prime}+{ }^{12} \mathrm{C}^{*}\left(1^{+} ; 15.1 \mathrm{MeV}\right)$. According to 70 ,

$$
f\left(\boldsymbol{q}^{2}\right)=\left[1-(1-\rho) / 6(b|\boldsymbol{q}|)^{2}\right]^{2} \exp \left[-\frac{1}{2}(b|\boldsymbol{q}|)^{2}\right]
$$

where $b=1.881 \pm 0.053 \mathrm{fm}$, and $\rho=0.23(1 \pm 0.1)$.

Using the EPT summarized above, Fukugita et al. [58] were able to determine the cross sections for eqs. (14)-(17) up to $E_{\nu} \sim 100 \mathrm{MeV}$ within $\sim 12 \%$ accuracies; these uncertainties reflect the ambiguities in the input data and isospin symmetry breaking in nuclei. Similar results were obtained by Mintz and Pourkaviani [59, 60]. As in the $\mathrm{A}=2$ case discussed earlier, the ratio of the $\mathrm{NC}$ cross section to the $\mathrm{CC}$ cross section is expected to be less affected by the nuclear physics ambiguities. When we vary the nuclear form factors within $\sim 10 \%$ (which are typical errors given in ref. [58]), $R \equiv$ $\sigma\left[{ }^{12} \mathrm{C}\left(\nu_{e}, \nu_{e}^{\prime}\right){ }^{12} \mathrm{C}^{*}\left(1^{+} ; 15.1 \mathrm{MeV}\right)\right] / \sigma\left[{ }^{12} \mathrm{C}\left(\nu_{e}, e^{-}\right)^{12} \mathrm{~N}(\right.$ gnd $\left.)\right]$ is found to change by less than $\sim 2$ $\%$.

We now remark on another semi-empirical method developed by Walecka and Donnelly to estimate the matrix elements of weak-interaction transitions in light nuclei. In the Walecka-Donnelly (W-D) method [71, 72, 21], one assumes that the weak-interaction transition operator can be approximated by a sum of single-particle multipole operators that arise in the impulse approximation and that the electomagnetic data can be used to place constraints on the nuclear matrix elements of these operators. Because of these basic assumptions it should be obvious that, contrary to the often-made claim, the W-D method is not a model-independent approach. Apart from the fundamental assumption pertaining to the impulse approximation, an important question is to what extent one can identify the 
effective matrix element of a single-nucleon operator $\mathcal{O}_{N}$ appearing in the weak-interaction process with that of the $\mathcal{O}_{N}$ that appears in the electromagnetic process. Chemtob and Rho [75] emphasized that the exchange-currents for the electromagnetic interaction can be drastically different from those of the weak processes. This point has been reemphasized in [43]. The EPT is free from these problems and hence, from a formal point of view, definitely a preferable approach. Having said this, we must quickly add that EPT in its rigorous form can be used at present only for the very limited case of $\mathrm{A}=12$. By contrast, the W-D method, owing to the additional assumptions made on the possible form of the transition operators, can be applied to many cases where the lack of necessary experimental information renders EPT at moment powerless. Thus, in practice, the W-D method is a very useful approach insofar as some independent information is available to check its reliability for given individual cases. The use of experimental data to constrain the parameters involved in the W-D method is in general expected to reduce nuclear-model dependence significantly, but the remaining possible uncertainties should be kept in mind.

Donnelly and Peccei [21] used the W-D method to estimate the cross sections for the NC reactions eqs. (14),(15). While a good agreement is seen between Donnelly and Peccei's results and those of Fukugita et al. [58] at lower incident energies, disagreement becomes apparent for $E_{\nu} \gtrsim 25 \mathrm{MeV}$, and it amounts to $60 \%$ at $\sim 50 \mathrm{MeV}$. Furthermore, while Fukugita et al. report an appreciable difference between ${ }^{12} \mathrm{C}\left(\nu, \nu^{\prime}\right){ }^{12} \mathrm{C}^{*}$ and ${ }^{12} \mathrm{C}\left(\bar{\nu}, \bar{\nu}^{\prime}\right)^{12} \mathrm{C}^{*}$ at higher energies due to the interference term $F_{A} F_{M}$, this behavior is not reproduced in [21]. These discrepancies seem to signal the breakdown of some assumptions involved in the W-D method [21] as applied to higher incident neutrino energies. On the other hand, for low neutrino energies, the EPT results justify the W-D method [71], in which the leading-order impulse-approximation transition matrix element $\langle\boldsymbol{\tau} \boldsymbol{\sigma}\rangle$ in the weak interaction process is deduced from the observed M1 strength. For ${ }^{12} \mathrm{C}\left(\nu_{e}, e^{-}\right){ }^{12} \mathrm{~N}$ the estimate of Fukugita et al. [58] is significantly smaller than the published result of the W-D method [73]; specifically, for the cross section averaged over the $\nu_{e}$ spectrum due to the $\mu$-decay, the EPT gives 
$<\sigma\left[{ }^{12} \mathrm{C}\left(\nu_{e}, e^{-}\right){ }^{12} \mathrm{~N}(\right.$ gnd $\left.)\right]>=9.2 \times 10^{-42} \mathrm{~cm}^{2}$, which is about $25 \%$ lower than that of Donnelly [73]. However, a more recent result of the W-D method [74] agrees with the EPT result.

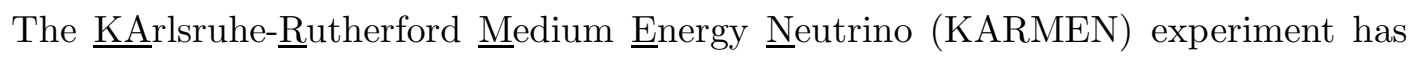
been intensively pursued at the spallation facility of the Rutherford Laboratory [56, 57, 76]. The pulsed beam dump neutrino source provides monoenergetic $\nu_{\mu}$ with $E_{\nu}=29.8 \mathrm{MeV}$ from $\pi^{+}$decay at rest as well as $\nu_{e}$ and $\bar{\nu}_{\mu}$ with energies up to $52.8 \mathrm{MeV}$ coming from the subsequent $\mu^{+}$decay. A 56 t liquid scintillation calorimeter [76 is used as massive live target of ${ }^{12} \mathrm{C}$. The $\mathrm{NC}$ reaction eq.(16) has been observed [77 by monitoring the $\gamma$ emission back to the ground state $\left(E_{\gamma}=15.1 \mathrm{MeV}, \mathrm{B} \cdot \mathrm{R}=94 \%\right)$. Experimentally [77], < $\sigma\left[{ }^{12} \mathrm{C}\left(\nu_{e}, \nu_{e}^{\prime}\right){ }^{12} \mathrm{C}^{*}\left(1^{+} ; 15.1 \mathrm{MeV}\right)\right]>^{\exp }=[9.5 \pm 1.8($ stat $) \pm 1.35$ (syst) $) \times 10^{-42} \mathrm{~cm}^{2}$, where $<\sigma>$ represents the average over the $\nu_{e}$ spectrum coming from the $\mu^{+}$decay. This agrees well with theoretical estimates $<\sigma\left[{ }^{12} \mathrm{C}\left(\nu_{e}, \nu_{e}^{\prime}\right)^{12} \mathrm{C}^{*}\left(1^{+} ; 15.1 \mathrm{MeV}\right)\right]>^{\text {theor }}=(9.9-10.3) \times 10^{-42} \mathrm{~cm}^{2}$ [58, 60, 62]. The ${ }^{12} \mathrm{C}\left(\nu_{e}, e^{-}\right)^{12} \mathrm{~N}$ (gnd) reaction can be identified by taking the delayed coincidence of the recoil $e^{-}$and the $e^{+}$from the ${ }^{12} \mathrm{~N} \beta$-decay. The KARMEN measurement [78, 89] gives $<\sigma\left[{ }^{12} \mathrm{C}\left(\nu_{e}, e^{-}\right)^{12} \mathrm{~N}(\right.$ gnd $\left.)\right]>^{\exp }=[8.0 \pm 0.75$ (stat) \pm 0.75 (syst) $] \times 10^{-42} \mathrm{~cm}^{2}$, which is consistent with the earlier Los Alamos result [53]: $\left\langle\sigma\left[{ }^{12} \mathrm{C}\left(\nu_{e}, e^{-}\right){ }^{12} \mathrm{~N}(\right.\right.$ gnd $\left.)\right]>^{\exp }=$ $[10.5 \pm 1.0($ stat $) \pm 1.0$ (syst) $] \times 10^{-42} \mathrm{~cm}^{2}$. These experimental values are in good agreement with recent theoretical estimates $\left\langle\sigma\left[{ }^{12} \mathrm{C}\left(\nu_{e}, e^{-}\right)^{12} \mathrm{~N}(\right.\right.$ gnd $\left.)\right]>^{\text {theor }}=(8.0-9.4) \times 10^{-42} \mathrm{~cm}^{2}$ given in [58, 74, 59].

EPT calculations on the $\nu_{-}{ }^{12} \mathrm{C}$ reactions for $E_{\nu} \gtrsim 100 \mathrm{MeV}$ were carried out by several authors. In particular, Mintz and Pourkaviani [61] extended the EPT calculation up to $E_{\nu}=3 \mathrm{GeV}$. The reliability of such extension, however, is not well established since the calculation involves the nuclear form factors at very high $q^{2}$, which are not known empirically except $F_{M}\left(q^{2}\right)$. There have also been attempts to apply EPT to nuclear systems other than the ${ }^{12} \mathrm{C}$ target [62] or to $\left(\nu_{\mu}, \mu\right)$ processes [59]. Here again, the problem is that there is not enough data to determine all relevant nuclear form factors empirically. The 
lack of experimental information necessitates the introduction of a number of additional theoretical assumptions, which are usually motivated by the impulse approximation results. This ad hoc procedure largely nullifies the predictive power of the original EPT.

Koetke et al. [54], using the in-flight pion decay neutrino source at LAMPF, measured the cross sections for ${ }^{12} \mathrm{C}\left(\nu_{\mu}, \mu^{-}\right) X$ and ${ }^{12} \mathrm{C}\left(\nu_{\mu}, \mu^{-}\right){ }^{12} \mathrm{~N}$ (gnd) at an average neutrino energy of $202 \mathrm{MeV}$. The cross section for the latter exclusive process is $\sigma\left[{ }^{12} \mathrm{C}\left(\nu_{\mu}, \mu^{-}\right)^{12} \mathrm{~N}(\text { gnd })\right]^{\exp }=$ $(1.7 \pm 0.8 \pm 0.3) \times 10^{-39} \mathrm{~cm}^{2}$. This is considerably higher than $0.8 \times 10^{-40} \mathrm{~cm}^{2}$ obtained in the W-D method 80, 81, 82 and an "EPT" estimate of $0.7 \times 10^{40} \mathrm{~cm}^{2}$ [59]. Here we have used the quotation mark since, as discussed above, a completely model-independent EPT analysis is not feasible in this case. According to Mintz and Pourkaviani [59], one can increase the predicted value sufficiently by enhancing the pseudoscalar form factor $f_{P}$ Although it is generally believed that the exchange-current effect quenches $f_{P}$ rather than enhances, there may be other competing nuclear effects. (For recent attempts to determine the effective $f_{P}$ inside nuclei using the radiative $\mu$-capture, see ref. 83 for experiment and ref. 84] for theory.) The inclusive reaction ${ }^{12} \mathrm{C}\left(\nu_{\mu}, \mu^{-}\right) X$ will be discussed later. 


\section{Chapter $4 \quad$ Empirical effective operator method (EEOM)}

Going back to the "realistic" nuclear Hamiltonian $H_{N}$ eq. (7), we recall that, even if we forgo the basic problem of deriving $H_{N}$ from the fundamental QCD and decide to be content with the operational usefulness of $H_{N}$, it is in general prohibitively difficult to determine true eigenstates $\Psi$ 's of $H_{N}$ and calculate the nuclear matrix elements of an observable $\mathcal{O}$ using $\Psi$ 's. This is true even when we limit ourselves to the nucleon-only regime, ignoring the exchange-current problem. One therefore usually splits the space subtended by $\Psi$ 's into two parts, a relatively manageable model space $P$ and the remainder $Q$, and calculate observables using wave functions $\Psi_{0}$ 's that belong to $P$. The phenomenological success of the shell model suggests choosing as $P$ the space of the lowest shell-model configurations; if necessary, slightly excited configurations can be included in $P$. Once the space of $\Psi$ 's is truncated to that of $\Psi_{0}$ 's, $H_{N}$ and $\mathcal{O}$ must undergo corresponding transformations, $H_{N} \rightarrow \tilde{H}_{N}$ and $\mathcal{O} \rightarrow \tilde{\mathcal{O}}$ so that $<\Psi^{\prime}\left|H_{N}\right| \Psi>=<\Psi_{0}^{\prime}\left|\tilde{H}_{N}\right| \Psi_{0}>$ and $<\Psi^{\prime}|\mathcal{O}| \Psi>=<\Psi_{0}^{\prime}|\tilde{\mathcal{O}}| \Psi_{0}>$ should hold. The nucleon-nucleon interactions that feature in $\tilde{H}_{N}$ are called the effective interactions, a somewhat historical terminology since from today's viewpoint the original $H_{N}$ itself is a highly "effective" entity. Similarly, $\tilde{\mathcal{O}}$ is called the effective operator. The difference between $\mathcal{O}$ and $\tilde{\mathcal{O}}$ represents the core-polarization effect. The formal framework to obtain the effective operators by incorporating effects of the eliminated space $Q$ does exist (for review, see [85, 86]), but applying this formalism to actual complex nuclei encounters many practical difficulties. Exceptionally, in the simplest cases of closed-shell nuclei plus or minus one nucleon, explicit calculations can be carried out to lowest orders of perturbation, as exemplified by the extensive work of Tower and Khanna 92, 93] and the University of Tokyo group (Arima, Shimizu, Hyuga et al) [94]. These authors calculated the magnetic moments, M1 transition strengths $B(\mathrm{M} 1)$, and the Gamow-Teller strengths $B(\mathrm{GT})$, taking into account up to second-order core polarization effects. Furthermore, the exchange-currents effects were carefully estimated and included in the final expressions of their effective operators. The results of these highly elaborate 
calculations are thoroughly documented in [93, 94].

Parallel to these theoretical attempts to derive the effective operators for the special cases, a useful semi-empirical approach which can cover a wider variety of nuclei has been developed by Wilkinson, Brown, Warburton and Wildenthal [87, 88, 89, 90, 91]. We shall refer to this approach as the empirical effective operator method (EEOM). As will be explained below, EEOM finds a useful application in estimating the cross sections of lowenergy neutrino-nucleus reactions for nuclei up to the $s d$-shell. We outline EEOM, taking the Gamow-Teller (GT) operator as an example 91].

Consider a GT transition $|i>\rightarrow| f>$ and suppose we are given reasonably realistic nuclear wavefunctions for $\mid i>$ and $\mid f>$. By "realistic" we usually mean the best available shell-model wavefunctions, which, as today's standard demands, are eigenvectors of a full matrix of $\tilde{H}_{N}$ covering all possible configurations within a given major shell. The shellmodel GT matrix element is given by

$$
\begin{aligned}
<G T> & \equiv<f ; J_{f}, T_{f}\left\|\mid \sum_{k=1}^{A} g_{A} \boldsymbol{\tau} \boldsymbol{\sigma}(k)\right\| \| i ; J_{i}, T_{i}>, \\
& =\sqrt{6} \sum_{j, j^{\prime}} D_{j^{\prime} j}<j^{\prime}\left\|g_{A} \boldsymbol{\sigma}\right\| j>
\end{aligned}
$$

where the summation over $j$ and $j^{\prime}$ goes over all the single-particle orbits contained in the model space $P$, and the one-body-transition density $D_{j^{\prime} j}$ is obtained from shell-model wavefunctions for an operator with rank in ordinary (isospin) space of $\Delta J(\Delta T)$ via

$$
D_{j^{\prime} j}=\frac{<J_{f}, T_{f}\left|\|\left[a^{\dagger}\left(j^{\prime}\right) \times \tilde{a}(j)\right]^{\Delta J, \Delta T}\right||| J_{i}, T_{i}>}{[(2 \Delta J+1)(2 \Delta T+1)]^{1 / 2}} .
$$

As stated, even if the model space $P$ is reasonably realistic, there are two significant sources of corrections to $\langle G T\rangle$, one from core polarization and the other from the exchangecurrent effect. If $P$ is large enough, the core polarization effect arises from rather highly excited states, and consequently its dependence on $\mid i>$ and $\mid f>$ will be weak. The same should hold for the exchange-current effect, which comes from the eliminated hadronic degrees freedom. In EEMO, therefore, one simulates these effects by replacing $\left.<j^{\prime}\left\|g_{A} \boldsymbol{\sigma}\right\| j\right\rangle$ 
in eq. (31) with an effective matrix element

$$
<j^{\prime}\left\|g_{A}^{\mathrm{eff}} \boldsymbol{\sigma}+g_{L A}^{\mathrm{eff}} \boldsymbol{\ell}+g_{P A}^{\mathrm{eff}}\left[Y_{2} \times \boldsymbol{\sigma}\right]^{(1)}\right\| j>
$$

while keeping $D_{j^{\prime} j}$ unchanged. This replacement will give an effective GT strength $<G T>>^{\text {eff }}$. To determine the effective coupling constants, $g_{A}^{\mathrm{eff}}, g_{L A}^{\mathrm{eff}}$ and $g_{P A}^{\mathrm{eff}}$ for a given major shell, one takes a sufficiently large number of GT decays that belong to this major shell and whose $f t$ values, or equivalently $B(\mathrm{GT})$ 's, are known experimentally. One then adjusts $g_{A}^{\text {eff }}, g_{L A}^{\text {eff }}$ and $g_{P A}^{\text {eff }}$ in such a manner that the $B(\mathrm{GT})$ 's calculated with $<G T>^{\text {eff }}$ would optimally reproduce the chosen set of experimental $B(\mathrm{GT})$ 's. Once $g_{A}^{\mathrm{eff}}, g_{L A}^{\mathrm{eff}}$ and $g_{P A}^{\mathrm{eff}}$ are determined, one can predict all other $B(\mathrm{GT})$ 's involving the same major shell.

The EEMO has proven to be highly successful in correlating a great majority of the observed magnetic moments, M1 strengths, and Gamow-Teller strengths over a wide range in the periodic table [89, 90, 91]. Furthermore, the effective coupling constants determined in EEOM show reasonable agreement with the corresponding quantities obtained theoretically for the closed-shell plus/minus one nucleon systems [93, 94].

Two p-shell nuclei, ${ }^{11} \mathrm{~B}$ and ${ }^{13} \mathrm{C}$, have recently been considered as useful targets to simultaneously monitor the NC and CC reactions for the solar neutrinos. In fact, Raghavan, Pakvasa and Brown's proposal 95] to use the ${ }^{11} \mathrm{~B}$ target is already being intensively pursued in the BOREX and BOREXINO projects. We describe here how the EEOM (or a closely related approach) was used to estimate the cross sections on these targets.

\section{1. ${ }^{13} \mathrm{C}$ target}

The possible advantage of a ${ }^{13} \mathrm{C}$-enriched scintillation counter as a solar-neutrino detector was suggested by Arafune et al. [96] and further studied in 98. These authors emphasized that the replacement of ${ }^{12} \mathrm{C}$ in a scintillator with ${ }^{13} \mathrm{C}$ not only makes the detector much more sensitive to $\mathrm{CC}$ reactions, but also allows efficient detection of the $\mathrm{NC}$ reactions, and that even the natural abundance of ${ }^{13} \mathrm{C}(\sim 1 \%)$ may be large enough to detect $\mathrm{NC}$ 
reactions in a large volume scintillator. The relevant reactions are the $\mathrm{CC}$ reaction

$$
{ }^{13} \mathrm{C}+\nu_{e} \rightarrow{ }^{13} \mathrm{~N}+e^{-}
$$

and the $\mathrm{NC}$ reaction

$$
{ }^{13} \mathrm{C}+\nu \rightarrow{ }^{13} \mathrm{C}^{*}+\nu^{\prime}
$$

For the solar neutrino energy region. only the following final states need to be considered [97. For the $\mathrm{CC}$ reactions, $\mid J^{\pi}=1 / 2^{-}, T=1 / 2($ gnd $)>, \mid 3 / 2^{-}, 1 / 2(3.51 \mathrm{MeV})>$, $\mid 1 / 2^{-}, 1 / 2(8.92 \mathrm{MeV})>$, and $\mid 3 / 2^{-}, 1 / 2(9.48 \mathrm{MeV})>$ in ${ }^{13} \mathrm{~N}$, with $Q=2.22 \mathrm{MeV}$ for the ground state transition. For the NC reactions, $\left|3 / 2^{-}, 1 / 2(3.68 \mathrm{MeV})>,\right| 1 / 2^{-}, 1 / 2(8.86 \mathrm{MeV})>$, and $\mid 3 / 2^{-}, 1 / 2(9.90 \mathrm{MeV})>$ in ${ }^{13} \mathrm{C}$.

The total cross section $\sigma$ for the CC reaction $\nu_{e}+\left|A>\rightarrow e^{-}+\right| B>$ is given in impulse approximation by

$$
\begin{aligned}
\sigma= & \frac{G_{F}^{2} \cos ^{2} \theta_{c}}{\pi} p_{e} E_{e} F\left(Z_{B}, E_{e}\right) \\
& \times \frac{1}{6\left(2 J_{A}+1\right)}\left[|<B\||\boldsymbol{\tau}|\| A>|^{2}+g_{A}^{2}\left|<B\left\||\boldsymbol{\tau} \boldsymbol{\sigma} \|| A>\left.\right|^{2}\right],\right.\right.
\end{aligned}
$$

where $g_{A}=-1.262$. The Fermi matrix element contributes only to a transition between isomultiplet members. One often uses the reduced transition strengths $B(\mathrm{~F})$ and $B(\mathrm{GT})$ defined as

$$
\begin{aligned}
B(\mathrm{~F}) & \left.\equiv \sum_{M_{B}, M_{A}}\left|<J_{B} M_{B}\right| t_{ \pm}\left|J_{A} M_{A}>\right|^{2}=\frac{1}{6}|<B||\boldsymbol{\tau}||| A\right\rangle\left.\right|^{2}, \\
B(\mathrm{GT}) & \equiv \sum_{M_{B}, M_{A}}\left|<J_{B} M_{B}\right| t_{ \pm} \boldsymbol{\sigma}\left|J_{A} M_{A}>\right|^{2}=\frac{1}{6}|<B||| \boldsymbol{\tau} \boldsymbol{\sigma}|||A>|^{2} .
\end{aligned}
$$

The NC cross section is given by

$$
\sigma=\frac{G_{F}^{2}}{\pi} g_{A}^{2}\left(E_{\nu}^{\prime}\right)^{2} \frac{1}{48}|<B||| \boldsymbol{\tau} \boldsymbol{\|}|| A>\left.\right|^{2} .
$$

For the $\mathrm{CC}$, if the $f t$ value of $\beta$ decay $|B>\rightarrow| A>$ is known, $\sigma$ is given modelindependently in terms of $f t$ :

$$
\sigma=\frac{2 \pi^{2} \ln 2}{m_{e}^{5} f t} p_{e} E_{e} F\left(Z_{B}, E_{e}\right) \frac{2 J_{B}+1}{2 J_{A}+1} .
$$


This applies to $\sigma\left[{ }^{13} \mathrm{C} \rightarrow{ }^{13} \mathrm{~N}\right.$ (gnd)]. From $\log (f t)^{\exp }=3.667 \pm 0.001$ for ${ }^{13} \mathrm{~N}($ gnd $) \rightarrow$ ${ }^{13} \mathrm{C}$ (gnd), we obtain model-independently $<\sigma\left[{ }^{13} \mathrm{C} \rightarrow{ }^{13} \mathrm{~N}(\right.$ gnd $\left.)\right]>=8.12 \times 10^{-43} \mathrm{~cm}^{2}$. Here $<\sigma>$ stands for the cross section averaged over the ${ }^{8} \mathrm{~B}$ neutrino spectrum. (Due to the threshold energy $E_{t h}=2.2 \mathrm{MeV}$, only the neutrinos from ${ }^{8} \mathrm{~B}$ decay are relevant here.) As ${ }^{13} \mathrm{~N}$ (gnd) $\rightarrow{ }^{13} \mathrm{C}$ (gnd) is a super-allowed transition, one can expect a strong feeding of ${ }^{13} \mathrm{~N}$ (gnd). For the transitions to the excited states in ${ }^{13} \mathrm{~N}$, for which no experimental $f t$ values are available, one must rely on theoretical estimations. The same applies to the NC reactions.

Fukugita et al. 98] used EEOM to calculate these cross sections. Thus, in eq. (37), $<B\||\boldsymbol{\tau} \boldsymbol{\sigma} \|| A>$ is replaced by

$$
<B\|\| \boldsymbol{\tau}\|\| A>^{\mathrm{eff}} \equiv<B\left\|\mid f_{A}^{\mathrm{eff}} \boldsymbol{\tau} \boldsymbol{\sigma}+f_{L A}^{\mathrm{eff}} \boldsymbol{\tau} \boldsymbol{\ell}+f_{P A}^{\mathrm{eff}} \boldsymbol{\tau}\left[Y_{2} \times \boldsymbol{\sigma}\right]^{(1)}\right\| \| A>
$$

Correspondingly, $B(\mathrm{GT})$ will change into $B(\mathrm{GT})^{\mathrm{eff}}$ [cf. eq.(39)]. (B(F) does not change because of charge conservation.) For a model space $P$, Fukugita et al. used the CohenKurath (CK) wave functions [99], which are full-p-shell wave functions. To determine the effective couplings, $f_{A}^{\text {eff }}, f_{L A}^{\text {eff }}$ and $f_{P A}^{\text {eff }}$, the following three ground-state transitions were used: ${ }^{15} \mathrm{O}\left(\beta^{+}\right){ }^{15} \mathrm{~N},{ }^{13} \mathrm{~N}\left(\beta^{+}\right){ }^{13} \mathrm{C}$, and ${ }^{11} \mathrm{C}\left(\beta^{+}\right){ }^{13} \mathrm{~B}$. Since $g_{L A}^{\text {eff }} \boldsymbol{\tau} \ell$ is known to be very small [94, 93], $f_{L A}^{\mathrm{eff}}$ was fixed at a commonly accepted value [94, 93]: $f_{L A}^{\mathrm{eff}}=0.011$. The best-fit values of the remaining parameters are $f_{A}^{\mathrm{eff}}=0.69, f_{P A}^{\mathrm{eff}}=0.19$. These effective coupling constants were used to calculate $<B\|\| \boldsymbol{\tau} \boldsymbol{\|} \mid A>^{\text {eff }}, B(\mathrm{GT})^{\mathrm{eff}}$, and the cross sections for the reactions eqs. (34), (35). The resulting $B(\mathrm{GT})^{\mathrm{eff}}$ 's were found to be smaller than the corresponding bare shell-model values by a factor $\sim 2$. According to Fukugita et al. [98], in the solar neutrino energy range, one needs to consider only the ground and first-excited states in ${ }^{13} \mathrm{~N}$ for the $\mathrm{CC}$ reactions, and the first excited state in ${ }^{13} \mathrm{C}$ for the NC reaction The calculated cross sections for the dominant reactions leading to the first excited states are: $\left\langle\sigma\left[{ }^{13} \mathrm{C} \rightarrow{ }^{13} \mathrm{~N}\left(3 / 2^{-}(3.51 \mathrm{MeV})\right)\right]>=2.62 \times 10^{-43} \mathrm{~cm}^{2}\right.$ for $\mathrm{CC}$, and $<\sigma\left[{ }^{13} \mathrm{C} \rightarrow{ }^{13} \mathrm{C}\left(3 / 2^{-}(3.68 \mathrm{MeV})\right)\right]>=1.16 \times 10^{-43} \mathrm{~cm}^{2}$ for $\mathrm{NC}$. 
Fukugita et al, comparing $B(\mathrm{GT}){ }^{\text {eff }}$ for ${ }^{13} \mathrm{C} \rightarrow{ }^{13} \mathrm{~N}\left(3 / 2^{-}[3.51 \mathrm{MeV}]\right)$ with the corresponding "empirical" value deduced from the $(p, n)$ reaction, assigned $\sim 30 \%$ uncertainty to the calculated $\sigma$. (The use of $(p, n)$ reactions to determine $B(\mathrm{GT})$ 's is discussed in chapter 5.) One could improve Fukugita et al's calculation significantly by using the results of a recent much more elaborate EEOM study [91]. Chou et al. 91] have carried out a comprehensive analysis of Gamow-Teller $\beta$ decays for the light $(A \leq 18)$ nuclei. The model space $P$ was taken to be either $(0 s)^{4}(0 p)^{A-4}$ or $(0 s)^{4}(0 p)^{A-4-n_{s d}}(1 s 0 d)^{n_{s d}}$. The effective GT operator was deduced for the $0 p$ shell from a least-squares fit to sixteen $B(\mathrm{GT})^{\exp }$ 's, with the results $f_{A}^{\mathrm{eff}} \approx 0.8, f_{L A}^{\mathrm{eff}} \approx 0$, and $f_{P A}^{\mathrm{eff}} \approx 0$. Using the deduced effective operator,

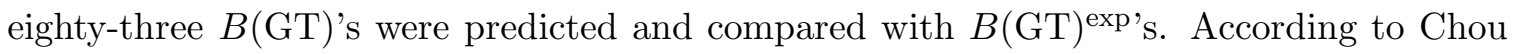
et al, their EEOM analysis can reproduce reasonably strong $B(\mathrm{GT})$ 's belonging to the $0 p$ shell within $\sim 10 \%$ accuracy. It may be worthwhile to redo the calculation of 98 taking into account Chou et al's latest result. In this connection one should also consult another detailed calculation on the p-shell nuclei due to the Utrecht group [101, 102], which utilized the full space of $0 \hbar \omega+2 \hbar \omega$ configurations.

According to Fukugita et al, the ${ }^{13} \mathrm{C}$ target is expected to have $6.22 \times(1 \pm 0.1) \mathrm{SNU}$ from the CC reactions, and $0.68 \times(1 \pm 0.3)$ SNU from the NC reaction. These correspond to $7870 \mathrm{CC}$ events per kiloton yr and $860 \mathrm{NC}$ events per kiloton yr for a ${ }^{13} \mathrm{CH}_{2}$ detector; these are both encouragingly large event rates.

The fact that number of final states that need to be taken into account for the ${ }^{13} \mathrm{C}$ detector is highly limited implies the possibility of direct calibration using neutrino beams from stopped muons. The cross sections averaged over the $\mu^{+}$-decay neutrino spectrum is estimated to be 98

$$
\begin{aligned}
<\sigma\left[{ }^{13} \mathrm{C}\left(\nu_{e}, e^{-}\right){ }^{13} \mathrm{~N}(\text { gnd })\right]>_{\mu^{+} \text {decay }} & =2.22 \times 10^{-41} \mathrm{~cm}^{2}, \\
<\sigma\left[{ }^{13} \mathrm{C}\left(\nu_{e}, e^{-}\right){ }^{13} \mathrm{~N}\left(\frac{3}{2}^{-} ; 3.51 \mathrm{MeV}\right)\right]>_{\mu^{+} \text {decay }} & =2.27 \times 10^{-41} \mathrm{~cm}^{2} \\
<\sigma\left[{ }^{13} \mathrm{C}\left(\nu_{e}, \nu_{e}\right){ }^{13} \mathrm{C}\left(\frac{3}{2}^{-} ; 3.68 \mathrm{MeV}\right)\right]>_{\mu^{+} \text {decay }} & =0.56 \times 10^{-41} \mathrm{~cm}^{2},
\end{aligned}
$$




$$
<\sigma\left[{ }^{13} \mathrm{C}\left(\bar{\nu}_{\mu}, \bar{\nu}_{\mu}\right)^{13} \mathrm{C}\left(\frac{3}{2}^{-} ; 3.68 \mathrm{MeV}\right)\right]>_{\mu^{+} \text {decay }}=0.74 \times 10^{-41} \mathrm{~cm}^{2}
$$

In this estimation, the finite momentum transfer effect is included by multiplying $B(\mathrm{~F})$ and $B(\mathrm{GT})^{\text {eff }}$ with $j_{0}(q r)$. This form factor effect reduces $\langle\sigma\rangle_{\mu^{+}}$decay by $\sim 15 \%$, but it is negligible $(<1 \%)$ for the solar neutrino energies. These estimates are also relevant to natural carbon scintillator experiments, where ${ }^{13} \mathrm{C}$ contamination may contribute a significant background.

4.2. ${ }^{11} \mathrm{~B}$ target - BOREX and BOREXINO experiments [95, 103, 104, 105, 106]

Borex is a boron-rich liquid scintillation detector that contains approximately $200 \mathrm{t}$ of boron in the fiducial volume, and is designed to measure the bolometric solar neutrino flux. Borex allows the observation of four basic reactions:

$$
\begin{aligned}
\nu+{ }^{11} \mathrm{~B} & \rightarrow \nu^{\prime}+\left[{ }^{11} \mathrm{~B}^{*}\left(E_{i}\right) \rightarrow \gamma\left(E_{x}\right)+{ }^{11} \mathrm{~B}\right], \\
\nu_{e}+{ }^{11} \mathrm{~B} & \rightarrow e^{-}+\left[{ }^{11} \mathrm{C}^{*}\left(E_{i}^{\prime}\right) \rightarrow \gamma\left(E_{x}^{\prime}\right)+{ }^{11} \mathrm{C}\right], \\
\nu+e^{-} & \rightarrow \nu^{\prime}+e^{-}, \\
\bar{\nu}_{e}+p & \rightarrow n+e^{+}
\end{aligned}
$$

The NC process involves the three excited states of ${ }^{11} \mathrm{~B}$ at $E_{x}=2.12,4.45$ and $5.02 \mathrm{MeV}$, while the $\mathrm{CC}$ process involves the four states in ${ }^{11} \mathrm{C}$ at $E_{x}^{\prime}=0,2.0,4.32$ and $4.80 \mathrm{MeV}$. The recent accumulation of solar neutrino experimental data as discussed in chapter 1 has enhanced the importance of measuring the solar neutrino flux coming from ${ }^{7} \mathrm{Be}$, and the fact that the ${ }^{11} \mathrm{~B}$ target has an appropriate detection threshold for this purpose further increases our interest in the BOREX project. These discussions can be found in [106]. Here we concentrate on the method used by Raghavan, Pakvasa and Brown in their celebrated 1986 paper [95] to estimate the cross sections for the nuclear processes eqs. (44), (45). The CC reaction cross section leading to the ${ }^{11} \mathrm{C}$ ground state can be directly connected to the experimental $f t$ value for the ${ }^{11} \mathrm{C} \rightarrow{ }^{11} \mathrm{~B} \beta$ decay [cf. eq. (41)] . To deduce the cross sections for the other final states, eq. (37) without the Fermi contribution is used. However, 
the magnitudes of quenching of the relevant $B(\mathrm{GT})$ 's are estimated using information on the electromagnetic process. That is, by exploiting the facts that the isovector part of the M1 operator also involves $\boldsymbol{\tau} \boldsymbol{\sigma}$ and that $B(\mathrm{M} 1)_{\mathrm{ISV}}^{\exp }$ 's between the ground state and the relevant excites states in ${ }^{11} \mathrm{~B}$ are known from $\gamma$ decay and $\left(e, e^{\prime}\right)$ data, the effective $B(\mathrm{GT})$ is determined by assuming the proportionality

$$
\frac{B(\mathrm{GT})^{\mathrm{eff}}}{B(\mathrm{GT})}=\frac{B(\mathrm{M} 1)_{\mathrm{ISV}}^{\mathrm{eff}}}{B(\mathrm{M} 1)_{\mathrm{ISV}}}
$$

This is in fact the Walecka-Donnelly (W-D) method [71, 72] discussed in the previous chapter rather than the EEOM as defined here. Although the core-polarization effect should quench $B(\mathrm{GT})$ and $B(\mathrm{M} 1)$ equally, the exchange-current effects on $B(\mathrm{GT})$ can be significantly different from those on $B(\mathrm{M} 1)$. The calculation of the exchange-current effect [93] indicates that the use of eq. (48) would introduce $\sim 30 \%$ errors. It is therefore in general preferable to use EEOM, in which $B(\mathrm{GT})^{\text {eff }}$ is deduced using only information on the weak-interaction processes. Since the effective GT operator for the p-shell based on the elaborate EEOM is now available [91], it seems warranted to derive new estimates of the cross sections relevant to BOREX with the use of EEOM. This will probably reduce the uncertainties in the cross sections from the $30 \%$ to $10 \%$ level. As far as the classification of calculational methods is concerned, we could have discussed ${ }^{11} \mathrm{~B}$ in relation to the W-D method. The reason why we discuss it in this chapter is the similarity of considerations that should go into ${ }^{13} \mathrm{C}$ and ${ }^{11} \mathrm{~B}$. 


\section{Chapter 5 Determination of Gamow-Teller strengths through $(p, n)$ reactions}

\subsection{Formalism}

The $(p, n)$ reaction has been used extensively to estimate the Gamow-Teller strengths $B(\mathrm{GT})$ that determine the cross sections of neutrino-nucleus reactions of great astrophysical importance. The key points here are that the $(p, n)$ reaction, in contrast to $\beta$ decay, is not limited by $Q$-value constraints, and that there exists an approximate "empirical" proportionality between $B(\mathrm{GT})$ and the forward $(p, n)$ reaction cross section at intermediate energies 107, 108, 109]. This proportionality was studied in great detail by Tadeucci et al. [109]. The essential points of their classic paper may be summarized as follows. Consider the $(p, n)$ reaction

$$
p\left(E_{p}\right)+{ }_{Z} A_{N} \rightarrow n\left(E_{n}\right)+{ }_{Z+1} A_{N-1}(j),
$$

where $j$ distinguishes various final states. The transition leading to state $j$ may be of the Fermi-, GT-, or mixed type; for the sake of simplicity, however, we first consider the pure cases of $\alpha$-type ( $\alpha=\mathrm{F}$ or GT), and describe later how to extend the results to the mixedtype transition. Let $\sigma_{j}^{\exp }\left(\mathrm{A}, E_{p} ; q, \omega\right)$ denote the observed differential cross section with $\omega \equiv$ $E_{p}-E_{n}, \boldsymbol{q}=$ three-momentum transfer, and $q=|\boldsymbol{q}|$. Since the $0^{\circ}$ cross section is particularly important, we introduce $\left.\sigma_{j}^{\exp }\left(0^{\circ} ; \omega\right) \equiv \sigma_{j}^{\exp }(q, \omega)\right|_{0^{\circ}}$, where the momentum transfer $q$ for $0^{\circ}$ is related to $\omega$ as $q=\omega / v_{p}$. For sufficiently high incident energies and for forward angles, the direct reaction mechanism should be dominant. Then, for a differential cross section $\sigma_{j}^{\text {DWIA }}\left(\mathrm{A}, E_{p} ; q, \omega\right)$ obtained in a DWIA calculation, we can expect $\sigma_{j}^{\text {DWIA }}(q, \omega) \approx$ $\sigma_{j}^{\text {exp }}(q, \omega)$. We therefore adopt as a basic ansatz the equality

$$
\sigma_{j}^{\exp }\left(0^{\circ} ; \omega\right)=\sigma_{j}^{\text {DWIA }}\left(0^{\circ} ; \omega\right)
$$

where $\left.\sigma_{j}^{\text {DWIA }}\left(0^{\circ} ; \omega\right) \equiv \sigma_{j}^{\text {DWIA }}(q, \omega)\right|_{0^{\circ}}$. In the direct-reaction picture, a set of quantum numbers corresponding to the isospin-flipping and/or spin-flipping of the incident nucleon is transferred in a single step to the target nucleus. This single-step transfer, which could be considered as arising from the exchange of mesons or meson-like effective particles, is 
completely analogous to what the electroweak current does to a nucleus. We therefore expect $\sigma_{j}^{\text {DWIA }}(q, \omega)$ for forward angles to be closely related to the corresponding $\beta$ decay strength $B(\alpha)$, where $\alpha=\mathrm{F}$ or GT depending on $j$.

To guide ourselves in quantifying this "close" relationship, we consider a simplified DWIA treatment which excludes orbital angular momentum transfer $(L=0$ contribution only), and in which the initial and final distortion effects are taken into account using the eikonal approximation [110]. This simplified treatment, to be referred to as the eikonal DWIA calculation, leads to an explicit proportionality relation 109]:

$$
\sigma_{j}^{\mathrm{Eik}}(q, \omega)=K\left(E_{p}, \omega\right) \exp \left[-x A^{1 / 3}+p(\omega)\right]\left|J_{\alpha}\right|^{2} \exp \left(-\frac{1}{3} q^{2}<r_{\alpha}^{2}>\right) B(\alpha)_{j}
$$

Although the actual deduction of $B(\alpha)_{j}$ always uses the $0^{\circ}$ cross sections, we are considering here general forward-angle scatterings, in which $q$ and $\omega$ are independent variables. In the present context it is useful to treat these two variables separately, since the $q$ - and $\omega$ dependence of the cross sections arises from quite different physical origins. The first factor in eq. (51) is kinematic in origin, and its explicit form is

$$
K\left(E_{p}, \omega\right)=\frac{E_{p} E_{n}}{\pi^{2}} \frac{k_{n}}{k_{p}}
$$

The second factor represents the effect of distortion of the initial and final nucleon waves, parametrized in terms of the square-well optical potentials $U_{i, f}=-\left(V_{i, f}+\mathrm{i} W_{i, f}\right)$ with radius $R=r_{0} A^{1 / 3} ; x=4 W_{i} r_{0} / v_{p}$ and $p(\omega)$ arises from the difference between the initial and final optical potentials. One could calculate $p(\omega)$ in the eikonal DWIA, but it will prove more useful to determine it empirically. $J_{\alpha}$ is, apart from a correction for the antisymmetrization of the valence nucleon and target nucleus, the volume integral of the $\mathrm{N}-\mathrm{N} t$-matrix in a channel corresponding to $\alpha$ :

$$
J_{\alpha}=\int V_{\alpha}(r) d^{3} r+(\text { Pauli-exchange term })
$$

where $V_{\alpha}(r)$ is the radial dependence of the relevant $t$-matrix; $t(1,2)=V_{\mathrm{F}}\left(r_{12}\right) \vec{\tau}_{1} \cdot \vec{\tau}_{2}$ or $t(1,2)=V_{\mathrm{GT}}\left(r_{12}\right) \vec{\tau}_{1} \cdot \vec{\tau}_{2} \vec{\sigma}_{1} \cdot \vec{\sigma}_{2}$. The fourth factor in eq. (51) accounts for the momentum- 
transfer dependence coming from the overall nuclear size effect as well as the $q$-dependence in the $L=0$ Bessel transform of $V_{\alpha}(r)$. We factor out the $q$ - and $\omega$-dependence in eq. (51) as

$$
\sigma_{j}^{\mathrm{Eik}}(q, \omega)=F^{\mathrm{Eik}}(q, \omega) \sigma_{j}^{\mathrm{Eik}}(q=0, \omega=0)
$$

where

$$
\begin{aligned}
F^{\operatorname{Eik}}(q, \omega) & \equiv \frac{\sigma_{j}^{\operatorname{Eik}}(q, \omega)}{\sigma_{j}^{\mathrm{Eik}}(q=0, \omega=0)} \\
& =\frac{K\left(E_{p}, \omega\right)}{K\left(E_{p}, \omega=0\right)} \exp \left(-\frac{1}{3} q^{2}<r^{2}>\right) \exp [p(\omega)-p(\omega=0)] .
\end{aligned}
$$

Similarly, we define

$$
F_{j}^{\text {DWIA }}(q, \omega) \equiv \frac{\sigma_{j}^{\text {DWIA }}(q, \omega)}{\sigma_{j}^{\text {DWIA }}(q=0, \omega=0)} .
$$

By definition, $F^{\text {Eik }}(q=0, \omega=0)=F_{j}^{\text {DWIA }}(q=0, \omega=0)=1$. The separability of $B(\alpha)_{j}$ in $\sigma_{j}^{\mathrm{Eik}}(q, \omega)[$ eq. (51)] implies that

$$
\hat{\sigma}_{j}^{\mathrm{Eik}}\left(A, E_{p}\right) \equiv \sigma_{j}^{\mathrm{Eik}}\left(A, E_{p} ; q=0, \omega=0\right) / B(\alpha)_{j},
$$

is independent of individual transitions $j$, except for the distinction between the $\mathrm{F}$ - and GT-types. We therefore write $\hat{\sigma}_{\alpha_{j}}^{\mathrm{Eik}}\left(A, E_{p}\right)$ instead of $\hat{\sigma}_{j}^{\mathrm{Eik}}\left(A, E_{p}\right)$. Its explicit form is

$$
\hat{\sigma}_{\alpha_{j}}^{\mathrm{Eik}}\left(A, E_{p}\right)=K\left(E_{p}, \omega=0\right) \exp \left[-x A^{1 / 3}+p(\omega=0)\right]\left|J_{\alpha_{j}}\right|^{2}
$$

We also introduce

$$
\hat{\sigma}_{j}^{\mathrm{DWIA}}\left(A, E_{p}\right) \equiv \sigma_{j}^{\mathrm{DWIA}}\left(A, E_{p} ; q=0, \omega=0\right) / B(\alpha)_{j} .
$$

The hatted quantities, $\hat{\sigma}_{\alpha_{j}}^{\mathrm{Eik}}\left(A, E_{p}\right)$ and $\hat{\sigma}_{j}^{\mathrm{DWIA}}\left(A, E_{p}\right)$, which represent $(p, n)$ cross sections one would obtain for $q=0, \omega=0$ if $B_{\alpha_{j}}=1$, are called the unit cross sections. In terms of the unit cross section $\hat{\sigma}_{\alpha_{j}}^{\mathrm{Eik}}\left(A, E_{p}\right)$, the separability encoded in eq. (51) is expressed as

$$
\sigma_{j}^{\mathrm{Eik}}(q, \omega)=F^{\mathrm{Eik}}(q, \omega) \hat{\sigma}_{\alpha_{j}}^{\mathrm{Eik}}\left(A, E_{p}\right) B(\alpha)_{j}
$$


Note that, since the way $B(\alpha)_{j}$ enters into $\hat{\sigma}_{j}^{\mathrm{DWIA}}\left(A, E_{p}\right)$ is yet to be determined, the definition in eq. (59) is nothing more than a convenient numerical normalization; no explicit expression such as eq. (58) can be derived therefrom..

Guided by eq. (60), we try to derive an approximate proportionality between $B(\alpha)_{j}$ and $\sigma_{j}^{\exp }\left(A, E_{p} ; q, \omega\right)$ or, more specifically, between $B(\alpha)_{j}$ and $\sigma_{j}^{\exp }\left(A, E_{p} ; 0^{\circ} ; \omega\right)$. The first step is to check to what extent the eikonal DWIA can reproduce the numerical results of the full DWIA calculation. For the purpose of comparing these two approximations concerning the reaction mechanism, one can use simplified nuclear wave functions. Tadeucci et al. [109], employing extreme single-particle wavefunctions, compared the eikonal DWIA and the full DWIA over a wide range of $A$ and $E_{p}$. Their results indicate that, if the parameters appearing in $F^{\mathrm{Eik}}(q, \omega)$ [eq. (55)] are treated as adjustable parameters, $F^{\mathrm{Eik}}(q, \omega)$ can fit $F^{\text {DWIA }}(q, \omega)$ reasonably well. The effective $F$ factor obtained through this fit is denoted by $F(q, \omega)$. Thus

$$
F(q, \omega) \equiv \frac{K\left(E_{p}, \omega\right)}{K\left(E_{p}, \omega=0\right)} \exp \left(-\frac{1}{3} q^{2}<\tilde{r}^{2}>\right) \exp [\tilde{p}(\omega)-\tilde{p}(\omega=0)]
$$

where $\tilde{r}$ is the optimal radial parameter, while $\tilde{p}(\omega)$ is the optimal functional form of $p(\omega)$. (Quadratic functions suffice to fit the DWIA results.) Furthermore, by fixing the parameters in eq. (58)] to best-fit $\hat{\sigma}_{j}^{\mathrm{DWIA}}\left(A, E_{p}\right)$, we can determine the optimized $\hat{\sigma}_{\alpha_{j}}^{\mathrm{Eik}}\left(A, E_{p}\right)$, which we denote by $\hat{\sigma}_{\alpha_{j}}\left(A, E_{p}\right)$. According to Tadeucci et al. [109, $\hat{\sigma}_{\alpha_{j}}\left(A, E_{p}\right)$ is a smooth function of $A$ and $E_{p}$ and is able to reproduce the average behavior of $\hat{\sigma}_{j}^{\text {DWIA }}\left(A, E_{p}\right)$ satisfactorily; for GT transitions $\left(\alpha_{j}=\mathrm{GT}\right)$, the scatter around $\hat{\sigma}_{\alpha_{j}}\left(A, E_{p}\right)$ due to the $j$-dependence is about $7 \%$. Using these results as well as eqs. (56), (59), we obtain

$$
\begin{aligned}
\sigma_{j}^{\text {DWIA }}(q, \omega) & =F_{j}^{\text {DWIA }}(q, \omega) \hat{\sigma}_{j}^{\text {DWIA }}\left(A, E_{p}\right) B(\alpha)_{j} \\
& \cong F(q, \omega) \hat{\sigma}_{\alpha_{j}}\left(A, E_{p}\right) B(\alpha)_{j}
\end{aligned}
$$

Since the various assumptions made in deriving this result are best justified for $0^{\circ}$ scattering, we specialize ourselves here to the $0^{\circ}$ case. Then, invoking the basic assumption eq. (50), 
we arrive at

$$
\left.\sigma_{j}^{\exp }\left(0^{\circ} ; \omega\right) \cong F(q, \omega)\right|_{0^{\circ}} \hat{\sigma}_{\alpha_{j}}\left(A, E_{p}\right) B(\alpha)_{j}
$$

Note that once the type of a transition $\left(\alpha_{j}=\mathrm{F}\right.$ or GT) is specified, the factor $\left.F(q, \omega)\right|_{0^{\circ}} \hat{\sigma}_{\alpha_{j}}\left(A, E_{p}\right)$ is independent of individual transitions specified by $j$, apart from the easily calculable kinematical dependence through $\omega$. Thus eq. (63) represents an approximate proportionality between $\sigma_{j}^{\exp }\left(0^{\circ} ; \omega\right)$ and $B(\alpha)_{j}$. The extension of this relation to the case of a mixed transition is

$$
\left.\sigma_{j}^{\exp }\left(0^{\circ} ; \omega\right) \cong F(q, \omega)\right|_{0^{\circ}} \times\left[\hat{\sigma}_{\mathrm{F}}\left(A, E_{p}\right) B(\mathrm{~F})_{j}+\hat{\sigma}_{\mathrm{GT}}\left(A, E_{p}\right) B(\mathrm{GT})_{j}\right]
$$

As a matter of fact, $F(q, \omega)$ slightly varies depending on $\alpha_{j}=\mathrm{F}$ or GT, through the $\alpha_{j^{-}}$ dependence of $\tilde{r}$ in eq. (61). This difference, however, is negligible in practice, and was ignored in the above expression.

In the literature one often encounters another expression which is equivalent to eq. (63). Dropping the optical potential in what is called here the eikonal DWIA, we consider the plane-wave impulse approximation (PWIA). The unit cross section in PWIA, $\hat{\sigma}_{j}^{\mathrm{PWIA}}\left(A, E_{p}\right)$, is given simply by ignoring the exponential factor in eq. (58):

$$
\hat{\sigma}_{j}^{\mathrm{PWIA}}\left(A, E_{p}\right)=K\left(E_{p}, \omega=0\right)\left|J_{\alpha_{j}}\right|^{2} .
$$

The ratio

$$
N^{D} \equiv \frac{\hat{\sigma}_{\alpha_{j}}^{\mathrm{Eik}}\left(A, E_{p}\right)}{\hat{\sigma}_{j}^{\mathrm{PWIA}}\left(A, E_{p}\right)}=\exp \left[-x A^{1 / 3}+p(\omega=0)\right],
$$

represents the optical potential effect for the $0^{\circ}$ scattering, and is called the distortion factor. Eqs. (57), (58) and (66) lead to

$$
\sigma_{j}^{\mathrm{Eik}}\left(A, E_{p} ; q=0, \omega=0\right)=K\left(E_{p}, \omega=0\right) N^{D}\left|J_{\alpha_{j}}\right|^{2} B(\alpha)_{j}
$$

This is completely equivalent to the $0^{\circ}$ value of eq. (60). Furthermore, upon optimizing the parameters appearing in eq. (67) to fit the full DWIA results, eq. (67) would become equivalent to eq. (63). 
The important question is how reliable eq. (63) is. As mentioned above, there is an intrinsic uncertainty $(\sim 7 \%)$ due to the scatter of $\sigma_{j}^{\text {DWIA }}(q, \omega)$ around the smooth function $\hat{\sigma}_{\alpha_{j}}\left(A, E_{p}\right)$. In fact, an even larger ambiguity arises from the optical-model dependence of $\sigma_{j}^{\text {DWIA }}(q, \omega)$ and $\hat{\sigma}_{\alpha_{j}}\left(A, E_{p}\right)$. These unit cross sections can vary up to $\sim 30 \%$ within reasonable choices of the optical potential. (The ratio $\sigma_{j}^{\text {DWIA }}(q, \omega) / \hat{\sigma}_{\alpha_{j}}\left(A, E_{p}\right)$ is not sensitive to this change.) Thus, at present, the proportionality coefficient in eq. (63) cannot be calculated with accuracy better than $\sim 30 \%$.

A useful alternative is to determine the proportionality coefficient empirically using transitions for which both $\sigma_{j}^{\exp }\left(0^{\circ} ; \omega\right)$ and $B(\alpha)_{j}^{\exp }$ are known. For each of these transitions one can deduce the empirical unit cross section via

$$
\left[\hat{\sigma}_{\alpha}^{\mathrm{emp}}\right]_{j} \equiv \frac{\sigma_{j}^{\exp }\left(0^{\circ} ; \omega\right)}{\left.F(q, \omega)\right|_{0^{\circ}} B(\alpha)_{j}^{\exp }} .
$$

If the proportionality relation eq. (63) is a good description of reality, $\left[\hat{\sigma}_{\alpha}^{\text {emp }}\right]_{j}$ 's should lie close to the smooth curve corresponding to $\hat{\sigma}_{\alpha_{j}}\left(A, E_{p}\right)$. For the GT process, Tadeucci et al. [109] studied a large number of transitions covering from $A=6$ to $A=162$ for various incident energies $E_{p}$. Tadeucci et al. refer to the proportionality among transitions with a common pair of initial and final nuclides as specific proportionality. The proportionality within transitions involving different pairs of nuclides is called general proportionality. Tadeucci et al.'s results indicate that the specific proportionality holds typically to an accuracy of $\sim 5 \%$. Thus the use of empirical data helps to reduce ambiguities which at present cannot be controlled theoretically. On the other hand, the same authors report that the general proportionality may be uncertain by as much as $\sim 50 \%$. In particular, the cases involving odd- $A$ targets exhibit the largest deviations from the predicted smooth curve. As far as the general proportionality is concerned, therefore, the model consideration described here is open to further improvements. The even-odd mass-number effect in the optical potential was cited as a possible solution for this problem 109].

The above argument suggests as a practical way to use the "proportionality" the 
following. To infer $B(\alpha)_{j}$ pertaining to a transition in a mass- $A$ nucleus, find a transition $j^{\prime}$ for which $B(\alpha)_{j^{\prime}}^{\exp }$ is known from a $\beta$-decay experiment and which belongs either to the same mass- $A$ nucleus or to the closest possible neighboring nucleus. Measure $\sigma_{j}^{\exp }\left(0^{\circ} ; \omega\right)$ and $\sigma_{j^{\prime}}^{\exp }\left(0^{\circ} ; \omega\right)$. Compute $\left[\hat{\sigma}_{\alpha}^{\mathrm{emp}}\right]_{j^{\prime}}$ from eq. (68). Obtain $B(\alpha)_{j}$ using eq. (63) together with the assumption $\hat{\sigma}_{\alpha_{j}}=\left[\hat{\sigma}_{\alpha}^{\mathrm{emp}}\right]_{j^{\prime}}$. This is in fact essentially the method that was used by Goodman et al. in their seminal paper 107], prior to the development of the detailed treatment described in [109].

As a particular case of the specific proportionality Tadeucci et al. also analyzed mixed-type transitions [eq. (64)] leading to the analog states of target nuclei. A very useful empirical rule to relate the $B(\mathrm{GT})$ of an analog transition to the $B(\mathrm{~F})$ in the same transition was reported in [109]. Note that $B(\mathrm{~F})$ is given model-independently by $B(\mathrm{~F})=N-Z$.

We add that all these results can also be stated using eq. (67), which is equivalent to eq. $(63)$.

\subsection{Experimental studies}

Systematic experimental studies of the GT-strength in nuclei with the use of intermediateenergy $(p, n)$ reactions dramatically started with two seminal articles in 1980 [111, 107]. The first paper [111] clearly demonstrated the existence of the giant GT resonance predicted by Ikeda, Fujii and Fujita as early as 1963 [112]. In the other paper [107, Goodman et al. [107] established the approximate proportionality eq. (63) between $B(\mathrm{GT})$ 's and the forward $(p, n)$ reaction cross section and emphasized that this proportionality could be a powerful tool to extract GT strengths for transitions inaccessible to $\beta$ decay. Indeed this tool has been used extensively in many subsequent works 108, 109, 113, 114, 115. Among the main objectives of these investigations are: (i) Determining GT-strength distributions over wide excitation-energy ranges and comparing them with shell-model predictions as well as with the GT-strength sum rule. (see e.g. 116, 115]); (ii) Extracting $B(\mathrm{GT})$ 's for particular transitions which play important roles in astrophysical neutrino detections. Although items (i) and (ii) are correlated, the latter is of more direct relevance to our discussion, and we 
mostly concentrate here on (ii).

A list of neutrino-nucleus reaction cross sections that are relevant to solar neutrino detection and that have been determined with the help of $(p, n)$ reaction data includes ${ }^{19} \mathrm{~F}$, ${ }^{37} \mathrm{Cl}$ [117], ${ }^{40} \mathrm{Ar},{ }^{71} \mathrm{Ga}$ 118], ${ }^{81} \mathrm{Br}$ [119, 124], ${ }^{98} \mathrm{Mo} \mathrm{[120],}{ }^{115} \mathrm{In},{ }^{127} \mathrm{I}$ 124], ${ }^{205} \mathrm{Tl}$ [121]. A thorough description of specific solar neutrino experiments that use these nuclear targets and a critical discussion of uncertainties in the estimates of the relevant cross sections can be found in Bahcall and Ulrich [4], and in Bahcall [1]. There is no need to repeat what is discussed extensively in these references but, in view of great current interest in the results of GALLEX 13] and SAGE [14, 15], it may be useful to remind the reader that the standard

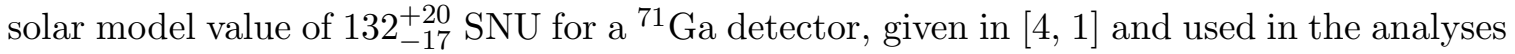
of the GALLEX and SAGE data, already includes rather generous error estimates of the $B(\mathrm{GT})$ 's for ${ }^{71} \mathrm{Ge}$ excited states. [The cross section for the ground-state transition can be determined from the $f t^{\exp }\left({ }^{71} \mathrm{Ge} \rightarrow{ }^{71} \mathrm{Ga}\right)$ without using the $(p, n)$ reaction, and hence has little uncertainty.]

We mention here two post-Bahcall-Ulrich developments. One is concerned with the

$$
\nu_{\mathrm{e}}+{ }^{127} \mathrm{I} \rightarrow \mathrm{e}^{-}+{ }^{127} \mathrm{Xe}(Q=0.789 \mathrm{MeV}) .
$$

Originally, Haxton [122] advocated the usefulness of an iodine detector for testing whether the solar neutrino flux is constant as a function of time. Our interest in the iodine detector has recently received an additional boost from the fact that it is sensitive to the ${ }^{7} \mathrm{Be}$ neutrinos as well as to the ${ }^{8}$ Be neutrinos. The results of the GALLEX and SAGE experiments [13, 14, 15] have further enhanced the importance of examining the energy dependence of solar neutrino deficits with the use of nuclear targets with different reaction $Q$-values. The ${ }^{127} \mathrm{I}$ target is a promising candidate to cover the region between the ${ }^{71} \mathrm{Ga}$ experiment and the water-Cerenkov-counter and ${ }^{37} \mathrm{Cl}$ experiments. The main issue with ${ }^{127} \mathrm{I}$ experiments is how to estimate the cross section of eq. (69) reliably, and this problem is currently studied with great intensity. [123]. For an update of $(p, n)$-reaction determination of the $B(\mathrm{GT})$ 's 
for ${ }^{127} \mathrm{I}$ as well as for ${ }^{71} \mathrm{Ga},{ }^{81} \mathrm{Br}$, see e.g. Sugarbaker [124]. For the current status of calibrating the ${ }^{127} \mathrm{I}\left(\nu_{e}, e^{-}\right){ }^{127} \mathrm{Xe}$ cross section with the LAMPF muon-decay neutrinos, see e.g. Wildenhain [125]; for the nuclear structure consideration of the relevant $B(\mathrm{GT})$ 's, see Engel et al. 126] and Haxton [127.

Another topic that has recently been attracting much attention will be discussed in the next section.

5.3 The low-energy $\nu_{e}+{ }^{37} \mathrm{Cl} \rightarrow \mathrm{e}^{-}+{ }^{37}$ Ar reaction

As is well known, the reaction

$$
\nu_{e}+{ }^{37} \mathrm{Cl} \rightarrow \mathrm{e}^{-}+{ }^{37} \mathrm{Ar},
$$

was the first to be used for detecting the solar neutrinos. It is also well known that the determination of the relevant cross sections presents a unique case. That is, there exists $\beta^{+}$ decay

$$
{ }_{20}^{37} \mathrm{Ca}_{17} \rightarrow{ }_{19}^{37} \mathrm{~K}_{18}+\mathrm{e}^{+}+\nu_{\mathrm{e}}
$$

which is the mirror process of

$$
{ }_{17}^{37} \mathrm{Cl}_{20} \rightarrow{ }_{18}^{37} \mathrm{Ar}_{19}+\mathrm{e}^{-}+\bar{\nu}_{\mathrm{e}}
$$

and all of the ${ }^{37}$ Ar states that are relevant to the solar neutrino reaction eq. (70) have their counterparts in the final ${ }^{37} \mathrm{~K}$ states of the ${ }^{37} \mathrm{Ca} \beta^{+}$-decay eq. (71), since this decay has a very large energy release $\left(Q_{\mathrm{EC}}=11.64 \mathrm{MeV}\right)$. Therefore, using the experimental $f t$-values for individual final states in eq. (71), one can determine model-independently low-energy cross sections for eq. (70) [cf. eq. (41)]. The mirror-asymmetry problem [128] can in principle affect this procedure to some extent. The mirror asymmetry parameter $\delta$ is defined by $\delta \equiv(f t)^{+} /\left(f t^{-}\right)-1$ for a pair of mirror $\beta^{+}$and $\beta^{-}$decays. With no charge-asymmetric effects in nuclear structure, and in the absence of second-class currents (SCC) in the weakinteraction current, $\delta$ should be identically zero. Experimentally, $\delta^{\exp } \lesssim 0.1$ for even- $A$ systems, and $\delta^{\exp }$ can be as large as $\sim 0.2$ for odd- $A$ nuclei 128. (It is commonly accepted 
that the finite values of $\delta^{\exp }$ are caused by the "trivial" nuclear charge-asymmetry effects rather than by the SCC; no explicit calculations that can explain the large $\delta^{\exp }$ in the odd-A cases have ever been presented, however [128, 129.) The $\delta$ effect should in principle exist in the $A=37$ case as well. Fortunately, however, the solar-neutrino reaction on ${ }^{37} \mathrm{Cl}$ is dominated by the feeding of the analog-state $\left(E_{x}=4.98 \mathrm{MeV}\right)$ whose strength is primarily governed by the well-known $B(\mathrm{~F})=N-Z$, a situation that renders the estimation of $\left.\sigma{ }^{37} \mathrm{Cl}\left(\nu_{e}, e^{-}\right){ }^{37} \mathrm{Ar}\right]$ significantly less sensitive to the mirror-asymmetry problem than the general magnitude of $\delta^{\exp }$ may suggest. As one goes beyond the solar neutrino energies, however, the contributions of the higher-lying states become progressively more important and hence the $\delta$ problem may acquire a practical significance.

Another problem whose importance increases as $E_{\nu}$ becomes larger than the solar neutrino energies is the discrepancy between the $B(\mathrm{GT})$ 's deduced from the ${ }^{37} \mathrm{Ca} \beta$ decay [130] and those obtained from the $(p, n)$ reaction [117. In discussing this problem, it is useful to consult the shell-model prediction [89] obtained with the use of the empirical transition operator method (cf. chapter 4) based on the Wildenthal wavefunctions 131. Now, while ${ }^{37} \mathrm{Ca} \rightarrow{ }^{37} \mathrm{~K}+\beta^{+}$data 130 give $B(\mathrm{GT})$ 's in fair agreement with the shell-model values for $E_{x} \leq 5.7 \mathrm{MeV}$, they yield too small $B(\mathrm{GT})$ 's for the levels in the $E_{x}=6.5-8.5 \mathrm{MeV}$ range. According to Adelberger and Haxton [132], this discrepancy arises because Sextro et al. [130] assumed that the delayed proton emission from ${ }^{37} \mathrm{~K}$ leads only to the $0^{+}$ground state of ${ }^{37} \mathrm{Ar}$, thus ignoring the expected substantial feeding of the $2^{+}(3.827 \mathrm{MeV})$ state; if the decay to this $2^{+}$state is properly taken into account, the resulting $B(\mathrm{GT})$ 's are rather close to the shell-model prediction. The $(p, n)$ data [117] indicate a strong (summed) $B(\mathrm{GT})$ in the $E_{x}=6.5-8.5 \mathrm{MeV}$ region in agreement with the shell-model prediction. The overall agreement between the $B(\mathrm{GT})$ 's obtained from the $(p, n)$ reaction [117] and the shell-model values [39] is reasonable except for levels in the vicinity of the analog state $\left(E_{x}=4.98\right.$ $\mathrm{MeV}$ ), for which the ${ }^{37} \mathrm{Cl}(\mathrm{p}, \mathrm{n}){ }^{37}$ Ar reaction data give too small $B(\mathrm{GT})$ 's. Adelberger and Haxton [132] suggested that this depletion of the GT strengths was caused by an incorrect 
subtraction procedure of the strong Fermi contribution.

García et al. 133 recently carried out a high-resolution, low-background experiment on ${ }^{37} \mathrm{Ca} \beta^{+}$decay, drastically improving the time-honored work of Sextro, Gough and Cerny [130]. For $E_{x} \leq 5.5 \mathrm{MeV}$, the $B(\mathrm{GT})$ 's obtained by García are in fair agreement with those of [130], except that García et al. see no evidence for feeding of a $4.679 \mathrm{Mev}$ level in ${ }^{37} \mathrm{~K}$. On the other hand, García et al. find much more strength at $E_{x} \geq 5.5 \mathrm{MeV}$, and the integrated $B(\mathrm{GT})$ is roughly twice that of Sextro et al. Furthermore, García et al. identify $\approx 50 \%$ more GT strength below $E_{x}=8.0 \mathrm{MeV}$ than was inferred from the $(p, n)$ data [117. According to Adelberger et al. [134], the integrated $B(\mathrm{GT})^{\exp }$ up to $E_{X}=8 \mathrm{MeV}$ does not require the renormalization of $g_{A}$ (see also [115]), and the quenching of $g_{A}$ indicated by shell-model analyses [90, 116] of the $\beta$ decay data may in fact suggest that the shell-model calculations [90, 116] tend to shift the $B(\mathrm{GT})$ 's to the lower excited levels. Since the $\beta$-decay data concerns only low-lying states, the shell-model fit of the observed $\beta$-decay strengths leads to the apparent quenching of $g_{A}$.

Critical examinations of various aspects of García et al.'s analyses 133, 134 have been made in [135, 136, 137]. The comparison of the latest ${ }^{37} \mathrm{Ca} \beta^{+}$decay result with the $(p, n)$ data points out among other things the following two problems: (i) On the $(p, n)$ side, the determination of the $B(\mathrm{GT})$ in the isobaric-analog state region $\left(E_{x} \approx 5 \mathrm{MeV}\right)$ is quite delicate because of the dominant Fermi transition and the limited experimental energy resolution [135]; (ii) On the $\beta$-decay side, the assumption used in 133 that all the ${ }^{37} \mathrm{~K}$ daughter states above the proton threshold should decay by proton emission is not tenable [136, 137]. In fact, the $3.24 \mathrm{MeV}$ level in ${ }^{37} \mathrm{~K}$ has $\Gamma_{\gamma} / \Gamma_{p} \approx 40$ [140], and this can change significantly García et al.'s analysis of the delayed-proton emission.

A detailed comparison between the updated values of $B(\mathrm{GT})^{\exp }$ and the improved shell-model prediction was made by Brown 139. The results of a recent re-measurement 138] of the ${ }^{37} \mathrm{Cl}(p, n){ }^{37} \mathrm{Ar}$ are expected to further clarify the issue.

It is to be remarked that, although from a nuclear-structure point of view it is im- 
portant and interesting to settle the existing quantitative difference between the ${ }^{37} \mathrm{Ca} \beta^{+}$ decay result and the $(p, n)$ data, the influence of this discrepancy on the solar neutrino cross section is rather modest; for instance, the $B(\mathrm{GT})$ 's deduced by García et al. 133 implies that $\sigma\left[{ }^{37} \mathrm{Cl}\left(\nu_{e}, e^{-}\right){ }^{37} \mathrm{Ar}\right]$ for the ${ }^{8} \mathrm{~B}$ neutrinos would be enhanced by $6 \%$ over the literature value. 


\section{Chapter $6 \quad$ Inclusive neutrino-nucleus reactions - Fermi gas model}

\subsection{Overview}

Our interest in intermediate- and high-energy neutrino-nucleus reactions was greatly enhanced by an intriguing issue raised by Davis [7]. According to Davis, the ${ }^{37}$ Ar production rate in the $\mathrm{Cl}$ experiment has a significant time structure which may be correlated with the occurrence of large solar flares [0]. A possible explanation is that, when protons accelerated by the flare activity collide with the solar atmosphere, they may produce a sufficient fluence of medium- and high-energy neutrinos (to be called the solar-flare neutrinos) to cause significant excess counts of ${ }^{37} \mathrm{Ar}$. The typical energies of the solar-flare neutrinos are $E_{\nu} \approx$ a few hundred $\mathrm{MeV}$, and their high energy tail can even extend to the $\mathrm{GeV}$ region. The original suggestion [7] was based on a crude estimate of the $\nu^{-37} \mathrm{Cl}$ cross section at $E_{\nu}=100 \mathrm{MeV}$, but quantitative considerations would require more elaborate estimates. Davis's argument based on the Homestake experiment was followed by the suggestion [2, 141, 142, 154] that large water Čerenkov detectors such as the Kamiokande and IMB facilities can furnish a powerful tool to measure the fluence of solar-flare neutrinos. The point is that because of the high energies of the flare-neutrinos the ${ }^{16} \mathrm{O}$ nuclei that are abundantly contained in a water detector become extremely efficient targets for detecting neutrinos via inclusive reactions [25]. A similar situation exists for the ${ }^{12} \mathrm{C}$ nuclei in scintillation counters [141]. These arguments call for reasonably reliable estimates of neutrino-nucleus reaction cross sections for $E_{\nu}$ typically up to a few hundred $\mathrm{MeV}$. Two different problems are involved here. For radio-chemical experiments that count only those events that lead to particlebound states, we must calculate semi-inclusive cross sections, whereas water Čerenkov or liquid scintillation experiments involve the usual inclusive reaction cross sections. Relegating the discussion of the semi-inclusive reactions to chapter 7 , we survey here recent works concerning the inclusive reactions.

The inclusive neutrino-nucleus reactions are also important in relation to the atmospheric neutrinos. When primary cosmic rays strike the atmosphere, they produce pions and 
kaons that subsequently decay into muons and muon-neutrinos, and much less frequently electrons and electron-neutrinos. The muons further decay into electron-neutrinos and muon-neutrinos. The neutrinos of this origin, called the atmospheric neutrinos, have been observed in the range of a few hundred $\mathrm{MeV}$ to several $\mathrm{GeV}$ with the large water Čerenkov detectors [143, 144]. The interactions of the atmospheric neutrinos in water are important for two main reasons. One is that these interactions constitute the principal background for nucleon decay experiments and extraterrestrial neutrino searches. The second reason is an important current issue concerning the atmospheric $\nu_{\mu} / \nu_{e}$ ratio. The above-described origin of the atmospheric neutrinos implies that there should be roughly two muon-neutrinos for each electron-neutrino. (Here "neutrinos" mean both neutrinos and antineutrinos.) The experimental data [143, 144], however, indicates that the muon-neutrinos are significantly less abundant than expected. The cross sections for ${ }^{16} \mathrm{O}\left(\nu_{\ell}, \ell^{-}\right) X$ (anything) and ${ }^{16} \mathrm{O}\left(\bar{\nu}_{\ell}, \ell^{+}\right) X$ (anything) feature importantly in analyzing these data.

\subsection{Calculational methods}

The study of inclusive nuclear responses to medium- or high-energy electroweak probes has a vast accumulation of literature [145, 71, 72, 146, 147, 148, 149, 151]. The closure approximation [146, 147] and the quasi-free scattering approach [71, 148, 149, 151] are the most commonly used methods. Kuramoto et al. [154, 155] have recently made a detailed comparison of these two formalisms and recommend that the closure approximation not be used for quantitative estimations. We therefore concentrate here on the quasi-free scattering approach.

We are concerned with the inclusive reactions

$$
\begin{aligned}
& \left.\nu_{\ell}+\mathrm{A} \rightarrow \ell^{-}+X \text { (anything }\right) \\
& \left.\bar{\nu}_{\ell}+\mathrm{A} \rightarrow \ell^{+}+X \text { (anything }\right) .
\end{aligned}
$$

In the quasi-free scattering (QFS) approach 145, 71 the transition amplitudes for the above processes are obtained by summing the transition amplitudes for neutrino-nucleon 
scattering over $A$ nucleons characterized by the initial and final nuclear wavefunctions. In the so-called relativistic treatment [148] one uses the original single nucleon amplitude given in terms of the Dirac spinors [30, 31]. In the non-relativistic approximation, one uses the non-relativistic (NR) reduction (the Foldy-Wouthuysen transformation) of the singleparticle transition amplitude [71].

In the NR approximation the cross sections for eqs. (73), (74) can be factorized into the single-nucleon cross section and the nuclear response function which accounts for the nucleon distribution inside nucleus and the Pauli blocking effect. Bugaev et al. 149] used the NR form truncated at first order in $|\boldsymbol{q}| / M$, where $\boldsymbol{q}$ is the three-momentum transfer. and calculated the inclusive cross sections on ${ }^{12} \mathrm{C},{ }^{16} \mathrm{O},{ }^{37} \mathrm{Cl},{ }^{71} \mathrm{Ga}$ and ${ }^{81} \mathrm{Br}$, for $E_{\nu} \lesssim 300$ $\mathrm{MeV}$. The nuclear wavefunctions were taken to be shell model wavefunctions in suitably chosen Woods-Saxon potential. Both bound and unbound orbits were allowed as the finalstate orbits. Gaisser and O'Connell [151] used the Fermi gas wavefunctions and calculated the inclusive cross sections for $E_{\nu}=50 \mathrm{MeV} \sim 2 \mathrm{GeV}$. To the extent that the Fermi gas model parameters $\left(p_{F}(p), p_{F}(n)\right.$, and the average nucleon separation energy $\left.E_{B}\right)$ can characterize actual nuclei, the results can be applied to any nuclear targets. Above the pion production threshold, $X$ in eqs. (73), (74) should include meson production processes, but they were not considered in [151]. (For neutrino-nucleus reactions leading to meson productions, see 152, 153].) According to Gaisser and O'Connell [151, (i) the relativistic and NR treatments give virtually identical results, (ii) in the energy region where their and Bugaev et al.'s calculations overlap, the results are similar, and (ii) the results are accurate to energies as low as $50 \mathrm{MeV}$ for electrons, and to threshold for muons. Thus, although Gaisser and O'Connell's calculation [151] has been playing an extremely important role in analyzing the $\nu_{\mu} / \nu_{e}$ ratio problem [143, 144], the measure of possible errors in the results is not readily available from 151.

Kuramoto, Fukugita, Kohyama and Kubodera [155] made a detailed study of the $\nu(\bar{\nu})-{ }^{16} \mathrm{O}$ inclusive reactions for $E_{\nu} \lesssim 300 \mathrm{MeV}$, using the relativistic Fermi gas (RFG) 
model of Smith and Moniz [148] as well as an improved non-relativistic Fermi gas (NRFG) model that incorporates the Foldy-Wouthuysen transformation up to third order in $|\boldsymbol{q}| / M$. One of the motivations for Kuramoto et al. to go beyond the lowest-order approximation of [151] is that in some kinematical regions the differential cross sections given in [151] become negative, a rather disturbing aspect. Kuramoto et al.'s NRFG treatment is found to be free from this problem and in reasonable agreement with the RFG model results. The NRFG cross sections systematically tend to be somewhat smaller than the RFG results and the difference, which grows with $E_{\nu}$, reaches $\sim 10 \%$ at $E_{\nu}=300 \mathrm{MeV}$. The agreement between the results of Kuramoto et al. and those of Bugaev et al. [149] is also at the $10 \%$ level.

For lower incident energies, there were attempts to add up explicitly the contributions of individual final nuclear states. Langworthy, Lamers and Überall (LLU) [156] considered eighteen final states for $\nu_{\ell}+{ }^{16} \mathrm{O} \rightarrow \ell^{-}+X$ (anything), and used the generalized Helm model to parametrize individual transition strengths, which were determined semi-empirically with the use of the electron scattering data. Haxton [25] carried out a summation of all possible final ${ }^{16} \mathrm{~F}$ states contained in a given truncated shell-model space; all configurations up to $2 \hbar \omega$ excitations were included for positive-parity final states and those up to $1 \hbar \omega$ excitations for negative-parity final states. As in the empirical effective operator method (cf. chapter 4) the shell-model transition rates were rescaled by adjustable multiplicative factors (up to $\sim 70 \%$ correction) so that the empirical $B(\mathrm{GT})$ values and the electron scattering data were reproduced. As discussed in Kuramoto et al. [155], $\sigma_{\text {Haxton }}$ agrees very well with $\sigma_{\mathrm{RFG}}$ for $E_{\nu} \lesssim 60 \mathrm{MeV}$ but starts to deviate appreciably beyond $E_{\nu} \approx 60 \mathrm{MeV}$; $\sigma_{\mathrm{LLU}}$ exhibits a similar tendency, but its deviation from $\sigma_{\mathrm{RFG}}$ beyond $E_{\nu} \approx 60 \mathrm{MeV}$ is more pronounced. Attributing these features to the fact that the LLU [156] took into account only eighteen final nuclear states whereas Haxton included much more, Kuramoto et al. argue that the difference between $\sigma_{\text {Haxton }}$ and $\sigma_{\mathrm{RFG}}$ for $E_{\nu} \gtrsim 60 \mathrm{MeV}$ is due to the limited size of the shell-model space used by Haxton. A later work of Haxton and Johnson [157] drastically extended the shell-model space and calculated the $\beta$-decay and $\mu$-capture. It would be 
interesting to see what cross section this extended calculation will give for the inclusive reactions, eqs. (73), (74).

Although it is gratifying that the RGF results smoothly join those of the explicit enumeration method around $E_{\nu} \approx 60 \mathrm{MeV}$, all these methods share the basic assumption that the quasi-free scattering picture is valid. The recent data on the transverse and longitudinal nuclear responses, $R_{T}$ and $R_{L}$, in quasi-elastic $\left(e, e^{\prime}\right)$ reactions indicate significant deviations from the Fermi-gas prediction even in the quasi-elastic peak region; the discrepancy can be as large as $\sim 20 \%$ for ${ }^{12} \mathrm{C}$ and $\sim 50 \%$ for ${ }^{40} \mathrm{Ca}$. Thus, the original remarkable success [158, 159, 160] of the Fermi-gas model in predicting the total (transverse +longitudinal) response needs to be taken with caution. There have been many attempts to explain $R_{T}$ and $R_{L}$, but a consistent picture is yet to be found [161]. The fact that we are dealing here with the total nuclear response is a favorable factor, but the fact that we are calculating the angle-integrated cross sections that include the non-quasi-elastic region may spoil to some extent the quasi-free scattering picture. Taking the mass-number dependence of the above-mentioned deviation from the Fermi gas model, Kuramoto et al. assigned $\sim 30 \%$ errors to their estimation of the total cross sections for $\nu_{\ell}+{ }^{16} \mathrm{O} \rightarrow \ell^{-}+X$ (anything) and $\bar{\nu}_{\ell}+{ }^{16} \mathrm{O} \rightarrow \ell^{+}+X$ (anything).

Singh and Oset 162 have recently made an extremely detailed study for ${ }^{12} \mathrm{C}$ and ${ }^{16} \mathrm{O}$, using another version of the Fermi gas model, the local Fermi gas model, which was tested in several other processes. Furthermore, these authors considered the nuclear-medium quenching of the weak-interaction effective coupling constants and the final-state Coulomb distortion effect. Comparison of Singh and Oset's results with those of Kuramoto et al. also indicates that the Fermi gas model is probably reliable up to the $\sim 30 \%$ level.

In actual experiments using a water Čerenkov detector, $\nu_{\ell}$ and $\bar{\nu}_{\ell}$ can also scatter from electrons. It is however easy to separate these events from $\nu(\bar{\nu})$ scattering off nuclei, since the former has a very sharp forward peak within $\theta \approx\left(m_{e} / E_{\nu}\right)^{1 / 2}$. Also, if the incident flux consists of a roughly equal mixture of $\nu_{e}$ and $\bar{\nu}_{e}$, the event rates of the reactions off 
oxygen nuclei will compete with that of $\bar{\nu}_{e}+p \rightarrow e^{+}+n$, and it is important to separate one from the other. This can also be done using the angular distribution [155].

The significance of the improved estimates of the cross sections for ${ }^{16} \mathrm{O}\left(\nu_{\ell}, \ell^{-}\right) X$ (anything) and ${ }^{16} \mathrm{O}\left(\bar{\nu}_{\ell}, \ell^{+}\right) X$ (anything) for the solar-flare neutrino problem will be discussed at the end of the next chapter. As far as the atmospheric neutrino problem is concerned, the question is to what extent the ambiguity in the Fermi gas model used in interpreting the data would affect the conclusion. Although one generally expects that a substantial part of the ambiguity will disappear upon taking the $\nu_{\mu} / \nu_{e}$ ratio [163], a more quantitative consideration seems warranted. 


\section{Chapter $7 \quad$ Semi-inclusive neutrino-nucleus reactions}

\section{1 .}

When high-energy neutrinos hit a nuclear target used in a radiochemical experiment, many nuclear levels can of course be excited but the experiment by design registers only the reactions leading to particle-stable states. If the number of final bound states is small enough we can hope to calculate the contributions of the individual levels and sum them up. (This might be the case for very light nuclei.) As $E_{\nu}$ gets higher, however, the possible final bound states can be too numerous for the explicit enumeration of the individual levels to be practical. We call this type of process the semi-inclusive reaction. Semi-inclusive reactions cannot be treated in the ordinary inclusive-reaction formalisms since particleunbound states must be excluded from the final states. A practically interesting example of semi-inclusive reactions was encountered when Davis [7] considered the reaction between the solar-flare neutrinos and the ${ }^{37} \mathrm{Cl}$ target:

$$
\nu_{\ell}+{ }^{37} \mathrm{Cl} \rightarrow \ell \ell^{-}+{ }^{37} \mathrm{~A} \quad(\ell=\mathrm{e} \text { or } \mu)
$$

where the final ${ }^{37} \mathrm{~A}$ stands for all particle-stable states. Davis's original argument used a rather crude estimate of this semi-inclusive cross section, which motivated Kuramoto et al. [154, 155 to make a more quantitative estimation. Although Kuramoto et al.'s treatment is highly specific to the $A=37$ system, we briefly describe that work here as a possibly useful example, since semi-inclusive reactions are likely to be relevant to other radiochemical experiments as well,

The general expression for cross section $\sigma$ for the reaction $\nu_{\ell}+\left|i>\rightarrow \ell^{-}+\right| f>$ is

$$
\sigma=(2 \pi)^{4} \sum_{f} \int d^{3} p_{\ell} \delta\left(E_{\ell}+E_{f}-E_{\nu}-E_{i}\right)\left|<\ell\left(p_{\ell}\right) ; f\right| H_{\mathrm{eff}}\left|\nu\left(p_{\nu}\right) ; i>\right|^{2} .
$$

The final nuclear states $\mid f>$ should lie below the neutron threshold energy, $E_{x}=8.8$ $\mathrm{MeV}$. In impulse approximation to be used here, $H_{\mathrm{eff}}$ is the sum of the single-particle weak-interaction operator. The precision of the calculation described here does not warrant 
taking account of the exchange current effects and other fine details. In this approximation

$$
\sigma=\frac{G^{2}}{\pi} \cos ^{2} \theta_{c} \sum_{f} p_{\ell} E_{\ell} F\left(Z, E_{\ell}\right) \frac{1}{2} \int_{-1}^{1} d(\cos \theta) M_{\beta},
$$

where $E_{\ell}=E_{\nu}-\Delta E_{f i}\left(\Delta E_{f i} \equiv E_{f}-E_{i}\right)$ is the outgoing lepton energy. The squared nuclear transition matrix elements $M_{\beta}$ is given by:

$$
M_{\beta}=M_{F}|<f| \tilde{1}|i>|^{2}+M_{G 0} \frac{1}{3}|<f| \tilde{\sigma}|i>|^{2}+M_{G 2} \Lambda
$$

where

$$
\begin{aligned}
& |<f| \tilde{1}|i>|^{2} \equiv \frac{1}{2 J_{i}+1} \sum_{M_{f}, M_{i}}\left|<f ; J_{f}, M_{f}\right| \sum_{k=1}^{A} t_{+}(k) e^{i \boldsymbol{q} \cdot \boldsymbol{r}_{k}}\left|i ; J_{i}, M_{i}>\right|^{2} \\
& =\frac{4 \pi}{2 J_{i}+1} \sum_{\ell}\left|<J_{f}\left\|\sum_{k} t_{+}(k) j_{\ell}\left(q r_{k}\right) Y_{\ell}\left(\hat{\boldsymbol{r}}_{k}\right)\right\| J_{i}>\right|^{2}
\end{aligned}
$$

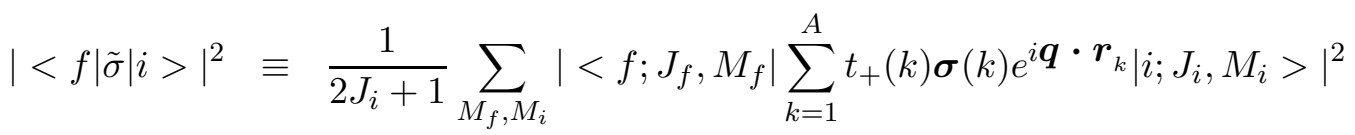

$$
\begin{aligned}
& =\frac{4 \pi}{2 J_{i}+1} \sum_{\ell, K}\left|<J_{f}\left\|\sum_{k} t_{+}(k) j_{\ell}\left(q r_{k}\right)\left[Y_{\ell}\left(\hat{\boldsymbol{r}}_{k}\right) \times \boldsymbol{\sigma}(k)\right]^{(K)}\right\| J_{i}>\right|^{2},
\end{aligned}
$$

and $\Lambda$ represents the interference between the $\langle f|\tilde{1}| i>$ and $\langle f|\tilde{\sigma}| i>$ terms. The coefficients, $M_{F}, M_{G 0}$ and $M_{G 2}$, contain the nucleon form factors as well as the kinematical quantities [155]. Now, of the transition operators appearing in eqs. (80), (82), the operators that satisfy the selection rule: " $\Delta J=0$ or $1, \Delta \pi=$ no" are classified as allowed-type operators. All the other operators are grouped together as forbidden-type. (This classification slightly differs from what is customarily used in $\beta$ decay theory.) In the present case with $|i>=|{ }^{37} \mathrm{Cl}(\mathrm{gnd}) ; \frac{3}{2}^{+}>$, the spin-parity of particle-bound states $\mid f>$ that can be reached via allowed-type operators is $\frac{1}{2}^{+}, \frac{3}{2}^{+}$, or $\frac{5}{2}^{+}$. The assembly of these states will be referred to as group A. Meanwhile, particle-bound states that can be fed only through the forbidden-type operators constitute group B, which contains negative-parity states as well as higher-spin positive-parity states.

For those levels of group A that predominantly belong to sd-shell configurations, reasonably realistic wave functions are available from the work of Wildenthal [131]. These 
wave functions are obtained by diagonalizing full sd-shell configurations and, with the use of the empirical effective operator method, the Wildenthal wave functions generally lead to a satisfactory agreement with the observed $B(\mathrm{GT})$ 's [131, 90]. The result of this detailed rescaling of the strengths can be simulated reasonably well by replacing the free-nucleon value $g_{A}=-1.262$ with an effective value $g_{A}^{\text {eff }} \approx-(0.7 \sim 1.1)$ [132]. At the time of Kuramoto et al.'s work, both the $B(\mathrm{GT})$ values determined from the ${ }^{37} \mathrm{Cl}(p, n)^{37}$ Ar reaction and those deduced from the ${ }^{37} \mathrm{Ca} \rightarrow{ }^{37} \mathrm{~K}+\beta^{+}$decay had some irregularities either among themselves or as compared with the shell-model predictions. Kuramoto et al. 155. used as the reference GT strengths the average of the $B(\mathrm{GT})$ values determined from these two methods, except that, for the $E_{x} \geq 6.5 \mathrm{MeV}$ region, they adopted the $(\mathrm{p}, \mathrm{n})$ data in view of the Adelberger and Haxton's argument 132 and, in the $E_{x} \approx 5 \mathrm{MeV}$ region, they used the $B(\mathrm{GT})$ 's obtained from the ${ }^{37} \mathrm{Ca} \beta$-decay with their strengths slightly reduced according to [132]. Comparison of this reference $B(\mathrm{GT})$ with the shell model prediction indicates that the shell model value (with $g_{A}^{\text {eff }} \approx-1.00$ ) slightly overestimates $B(\mathrm{GT})$ for $E_{x}<4 \mathrm{MeV}$ (by a factor of 1.38 on the average) and slightly underestimate it for $E_{x}>4 \mathrm{MeV}$ (by a factor of 0.78 ). In order to reproduce $B(\mathrm{GT})$ for $E_{x}<4 \mathrm{MeV}$, one needs to use $g_{A}^{\text {eff }}=-0.85$. Kuramoto et al. assumed that this coupling constant could be used universally for the whole range of $E_{x}$. This implies that the average shell-model value of $B(\mathrm{GT})$ for $E_{x}>4 \mathrm{MeV}$ is smaller than the experimental value by a factor of $0.78 \times 0.72=0.56$. This deficit was interpreted as representing the contribution of configurations outside the sd-shell. Using this information on $B(\mathrm{GT})$, Kuramoto et al. calculated $\sigma_{A}$ as

$$
\sigma_{A}=\sigma_{\text {analog }}+\sum_{E_{x} \leq 4 \mathrm{MeV}}+\alpha \sum_{4<E_{x}<8.8 \mathrm{MeV}}
$$

where all the axial-vector contributions were calculated for the Wildenthal wave functions using $g_{A}^{\text {eff }}=-0.85$, and $\alpha \equiv \frac{1}{0.56}=1.77$ was introduced to "recover" missing contributions of the outside-sd-shell contributions.

The levels belonging to group B may be divided into two subgroups: group $B_{s d}$ con- 
sisting of positive-parity states with $J_{f} \geq \frac{7}{2}$ which belong to the sd-shell, and group B' that comprises all others. The contribution of group $B_{s d}$, denoted by $\sigma_{B}^{s d}$, is estimated using the Wildenthal wave functions and $g_{A}^{\text {eff }}=-0.85$. The contribution of group B', denoted by $\sigma_{B^{\prime}}$, is assessed by using $\sigma_{B^{\prime}}=\sigma^{\text {forbid }} N_{B^{\prime}}$, where $\sigma^{\text {forbid }}$ is an estimate of the typical cross section to excite a level of group B', and $N_{B^{\prime}}$ the estimated number of levels belonging to group B'. The $\sigma^{\text {forbid }}$ was estimated by taking the average of $\sigma_{B}^{s d}$ and $\left(\sigma_{A^{\prime}}^{s d}\right)_{\text {forbid }}$, where $\left(\sigma_{A^{\prime}}^{s d}\right)_{\text {forbid }}$ is defined through the decomposition $\sigma_{A^{\prime}}^{s d}=\left(\sigma_{A^{\prime}}^{s d}\right)_{\text {allowed }}+\left(\sigma_{A^{\prime}}^{s d}\right)_{\text {forbid }}$, with $\left(\sigma_{A^{\prime}}^{s d}\right)_{\text {allowed }}\left[\left(\sigma_{A^{\prime}}^{s d}\right)_{\text {forbid }}\right]$ being the contribution of the allowed-type [forbidden-type] operators. The assessment of $N_{B^{\prime}}$ is a delicate job, but referring to the available information [164] on the level structure in ${ }^{37} \mathrm{Ar}$, Kuramoto et al. estimated $N_{B^{\prime}} \approx 28 \sim 34$.

The total contribution is obtained as

$$
\sigma=\sigma_{A}+\sigma_{B}=\sigma_{A}+\sigma_{B}^{s d}+\sigma_{B^{\prime}}
$$

Using this expression, Kuramoto et al. [155] calculated the cross section for the semiinclusive process eq. (75). The cross section rises up to $150 \sim 200 \mathrm{MeV}$ and then levels off. This is a generally expected behavior for a semi-inclusive reaction feeding bound states, since the growth of the phase space factor is counterbalanced by a suppression due to the nuclearsize effect. Kuramoto et al. estimated the error of their results to be $20 \%$ at $50 \mathrm{MeV}, 40-50$ $\%$ at $100 \mathrm{MeV}$, and 50-60\% or more at $150 \mathrm{MeV}$. The cross section for $E_{\nu}>150 \mathrm{MeV}$ may be subject to a factor 2 uncertainty. For the use in calibration experiments with a muon beam, the cross section averaged over the neutrino-spectrum from the stopped muon decay is relevant. Kuramoto et al. 1155 give $\sigma\left({ }^{37} \mathrm{Cl}\right)_{\mu^{+}-\text {decay }}=(9.4 \pm 1.4) \times 10^{-41} \mathrm{~cm}^{2}$. This value should be compared with $9.90 \times 10^{-41} \mathrm{~cm}^{2}$ given in [165], and with $7.6 \times 10^{-41} \mathrm{~cm}^{2}$ 㧮.

Although the method used by Kuramoto et al. is far from systematic, it is at present difficult to do better. It is hoped that the progress of main-stream shell model calculations will eventually reach the stage wherein the adopted model space is sufficiently large to 
accommodate all important final states of semi-inclusive neutrino-nucleus reactions, at least up to $s d$-shell nuclei. These shell-model model wavefunctions plus a judicious use of the empirical effective operator method (chapter 5) will probably allow us to treat the semiinclusive reactions in a more systematic way. For nuclear targets beyond the $s d$-shell, the possibility of such a systematic treatment seems rather remote.

\subsection{Consequences for the solar-flare problem}

Kuramoto et al. [155] used their estimate of the semi-inclusive reaction eq. (75) to deduce the solar-flare neutrino flux corresponding to the fifty excess ${ }^{37} \mathrm{Ar}$ events in the 615 ton $\mathrm{C}_{2} \mathrm{Cl}_{4}$ Homestake detector; this excess was assumed by Davis [7] to be of the solar-flare origin. For the flare-neutrino spectrum, a soft neutrino spectrum of the form $\phi\left(E_{\nu}\right)=\phi_{0} \exp \left(-E_{\nu} / E_{0}\right)$, with $E_{0}=50 \mathrm{MeV}$, as well as a monoenergetic form with various plausible energies was used. Based on the solar-flare neutrino flux deduced therefrom and their estimate (chapter 5) of the cross section for ${ }^{16} \mathrm{O}\left(\nu_{\ell}, \ell^{-}\right) \mathrm{X}$ (anything), Kuramoto et al. calculated the number of neutrino events to be expected in the Kamiokande detector during the solar flare periods and concluded that the observed counting rate of the neutrino events [143] fell far below the prediction. This corroborates the earlier conclusion of the Kamiokande group [142], that the time-integrated "solar-flare $\nu_{e}$ " flux is less than $3.7 \times 10^{7} \mathrm{~cm}^{-2}$ per flare for $E_{\nu}=100 \mathrm{MeV}$, which is 2000 times smaller than the value required to account for the excess "signal" observed in the ${ }^{37} \mathrm{Cl}$ experiment. Thus, the role of the solar-flare neutrinos in giving rise to the time structure in the Homestake solarneutrino data must be much smaller than described in [7]. For further detailed studies on the statistical significance of the time structure in the solar-neutrino data, see [166, 167, 168]. The untenability of Davis's original suggestion does not diminish the significance of using water Čerenkov counters, like the Kamiokande or IMB, to monitor intermediate energy neutrinos associated with the solar activity. This will allow us to explore the active Sun with a new, unconventional means, and also to have a better understanding of the particle 
acceleration mechanism around the solar sphere. It is to be emphasized that the sensitivity of the Kamiokande and IMB water detectors reaches the flux level of an optimistic estimate for a large flare or for more constant activity during the solar maximum period [169, 170]. 


\section{Chapter $8 \quad$ Additional remarks}

In the foregoing chapters we have discussed various nuclear physics aspects that go into the estimation of neutrino-nucleus reactions of astrophysical interest. Neutrinonucleus reactions can also be useful in studying the structure and symmetry properties of the fundamental coupling of the weak current with matter. In some of these experiments nuclei serve just as an assembly of $A$ nucleons, whereas in many others one takes advantage of a wide choice of selection rules pertaining to specific nuclear targets.

An example of the former is the recent determination of a stringent upper limit to the second-class current by Ahrens et al. [171]. These authors measured quasielastic reaction $\bar{\nu}_{\mu} p \rightarrow \mu^{+} n$ occurring in the scintillation target detectors for $q^{2}$ up to $1.0(\mathrm{GeV} / c)^{2}$. For the second-class axial-vector current strength defined by

$$
<n\left(p_{f}\right)\left|A_{\mu}^{\mathrm{SCC}}\right| p\left(p_{i}\right)>=\mathrm{i} \frac{f_{T}}{M} \bar{u}\left(p_{f}\right) \gamma_{5} \sigma_{\mu \nu} q^{\mu} u\left(p_{i}\right)
$$

with $q \equiv p_{f}-p_{i}$, and for the dipole parametrization of the $q^{2}$-dependence $f_{T}\left(q^{2}\right)=$ $\eta f_{A}(0) /\left(1+q^{2} / M_{T}^{2}\right)^{2}$, Ahrens et al. deduced $|\eta| \leq 0.8,0.23,0.12$ for $M_{T}\left(\mathrm{GeV} / \mathrm{c}^{2}\right)=$ $0.5,0.9,1.5$, respectively. These upper limits are substantially lower than the value obtained from analyzing the totality of the existing $\beta$ decay data. [172, 66]. To the extent that the incoherent sum of the individual quasielastic scattering off a nucleon provides an accurate description of the process, no nuclear dynamics is involved here. Although this picture is likely to be valid, it is probably worthwhile to check its validity in a quantitative manner in view of the breakdown (up to 30\%) of the quasi-free scattering assumption in the longitudinal and transverse response functions in $\left(e, e^{\prime}\right)$ processes.

There have been numerous attempts at exploiting various selection rules available from specific nuclear quantum numbers to study unconventional pieces of the weak current. Although it is beyond the scope of this review to discuss them in detail, we mention here as an example a possible consequence of the strangeness content of the nucleon for neutrinonucleus reactions. Although this effect will not change drastically the interpretation of 
astrophysical neutrino-nucleus reactions, it has its own fundamental importance.

The strange quark matrix elements of the nucleon are one of the central issues in contemporary particle-nuclear physics. The measurement of polarized deep-inelastic muon scattering on the proton [173], combined with an analysis of hyperon $\beta$-decay [174], suggests that there is a large contribution of the strange sea quarks $(\bar{s} s)$ to the proton spin. The pion-nucleon sigma term $\Sigma_{\pi N}$ also indicates a significant amplitude of $\langle N|\bar{s} s| N\rangle$, although the latest estimate [176] of $\Sigma_{\pi N}$ favors somewhat smaller $\bar{s} s$ content than in [175]. Beise and McKeown [177] have discussed parity violation measurements of polarized electronproton scattering to study strange quark matrix elements of the vector current. If such a large $\bar{s} s$ component exists in the nucleon, one should expect a sizable contribution also in the neutrino-nucleus reactions. In fact, neutrino neutral-current reactions offer the best means to study the strange quark matrix element of the axial current, which complement the electron-scattering experiments that are sensitive only to the vector current.

In the presence of the strange quarks, the hadronic neutral current $J_{\mu}^{3}$ appearing in eq. (18) would become $J_{\mu}^{\mathrm{NC}}$, where

$$
J_{\mu}^{\mathrm{NC}}=J_{\mu}^{T=1}+J_{\mu}^{T=0}
$$

with the isovector and isoscalar pieces defined by

$$
\begin{aligned}
& J_{\mu}^{T=1}=\left(1-2 \sin ^{2} \theta_{W}\right) V_{\mu}^{3}+A_{\mu}^{3} \\
& J_{\mu}^{T=0}=-2 \sin ^{2} \theta_{W} V_{\mu}^{0}+V_{\mu}^{s}+A_{\mu}^{s} .
\end{aligned}
$$

The standard forms for the effective isovector currents in the nucleon sector are

$$
\begin{aligned}
V_{\mu}^{(3)} & =\bar{u}_{N^{\prime}}\left(F_{1}^{V} \gamma_{\mu}+F_{2}^{V} \frac{i \sigma_{\mu \nu} q^{\nu}}{2 m_{N}}\right) \frac{\tau_{3}}{2} u_{N} \\
A_{\mu}^{(3)} & =g_{A} \bar{u}_{N^{\prime}} \gamma_{\mu} \gamma_{5} \frac{\tau_{3}}{2} u_{N},
\end{aligned}
$$

where $F_{1}^{V}$ and $F_{2}^{V}$ are isovector form factors, and $g_{A}(0)=-1.26$. Similarly, the isoscalar and the strange quark currents [177] are expressed as

$$
V_{\mu}^{(0)}=\bar{u}_{N^{\prime}}\left(F_{1}^{0} \gamma_{\mu}+F_{2}^{0} \frac{i \sigma_{\mu \nu} q^{\nu}}{2 m_{N}}\right) \frac{1}{2} u_{N}
$$




$$
\begin{aligned}
V_{\mu}^{(s)} & =-\frac{1}{2} \bar{u}_{N^{\prime}}\left(F_{1}^{s} \gamma_{\mu}+F_{2}^{s} \frac{i \sigma_{\mu \nu} q^{\nu}}{2 m_{N}}\right) \frac{1}{2} u_{N} \\
A_{\mu}^{(s)} & =\frac{1}{2} G_{1}^{s} \bar{u}_{N^{\prime}} \gamma_{\mu} \gamma_{5} \frac{1}{2} u_{N},
\end{aligned}
$$

where $F_{1}^{0}$ and $F_{2}^{0}$ are isoscalar form factors, and $F_{1}^{s}, F_{2}^{s}$ and $G_{1}^{s}$ denote the strange quark form factors of the nucleon for the vector current and for the axial vector current, respectively. Attempts to extract the strange quark axial form factor $G_{1}^{s}(0)$ have been made using both EMC [173, 174] and $\nu-p$ scattering data [178, 179]. The extracted form factors are

$$
G_{1}^{s}(0)= \begin{cases}-0.38 \pm 0.11 & \text { from EMC } \\ -0.30 \pm 0.16 & \text { from } \nu-p .\end{cases}
$$

However, it should be emphasized that the quoted numbers are not yet well established, because of several assumptions made in the analyses [179], so that the quoted errors are the experimental ones only. An interesting question is to what extent neutrino-nucleus reactions can sharpen our conclusion on the strange-quark neutral current form factors. Henley et al. [180] have investigated the asymmetry between the elastic cross-sections for $\nu+d \rightarrow \nu+d$ and $\bar{\nu}+d \rightarrow \bar{\nu}+d$. In impulse approximation, the difference in cross sections is given by

$$
\begin{aligned}
N \equiv & \frac{1}{2}\left\{\frac{d \sigma}{d q^{2}}(\nu d \rightarrow \nu d)-\frac{d \sigma}{d q^{2}}(\bar{\nu} d \rightarrow \bar{\nu} d)\right\} \\
& =\frac{4}{3} \frac{G_{F}^{2} m_{N}}{\pi E} \eta(1-\zeta) G_{1}^{s}\left(F_{1}^{0}+F_{2}^{0}\right) F_{D}
\end{aligned}
$$

where $\eta=q^{2} / 4 m_{N_{\nu}}^{2}, \zeta=q^{2} / 8 m_{N} E_{\nu}$, with $q$ the momentum transfer. $E_{\nu}$ is the neutrino (anti-neutrino) energy, and $F_{D}$ is the deuteron form factor. According to Henley et al.'s calculation the asymmetry $R$ defined by

$$
R \equiv \frac{\left(d \sigma / d q^{2}\right)(\nu d \rightarrow \nu d)-\left(d \sigma / d q^{2}\right)(\bar{\nu} d \rightarrow \bar{\nu} d)}{\left(d \sigma / d q^{2}\right)(\nu d \rightarrow \nu d)+\left(d \sigma / d q^{2}\right)(\bar{\nu} d \rightarrow \bar{\nu} d)}
$$

could be as large as $15 \%$ for large $q^{2}\left(\geq 0.5 \mathrm{GeV}^{2} / c^{2}\right)$. Suzuki, Kohyama and Yazaki 181 made a detailed study of low-energy $\nu{ }^{12} \mathrm{C}$ and $\nu \_{ }^{10} \mathrm{~B}$ reactions to extract information on $G_{1}^{s}$. Bernabéu et al. [182] studied the effect of the strange quark matrix element for the neutral-current reaction

$$
\nu+{ }^{11} \mathrm{~B} \rightarrow \nu+{ }^{11} \mathrm{~B}^{*}
$$


This reaction is particularly interesting, because it is directly related to BOREX or BOREXINO experiment [95]. It is to be noted that, unlike the $\nu d$ reactions, the isovector and isoscalar currents contribute coherently to the cross section here. A similar situation should exist for the $\nu^{13} \mathrm{C}$ neutral-current reaction discussed in chapter 4.

The above considerations were all based on the impulse approximation. Very recently, Hjorth-Jensen, Kirchbach, Riska and Tsushima [183] have pointed out that the exchange currents can make the effective isoscalar axial current density of a nucleon in a nucleus considerably different from the corresponding free nucleon value. The isoscalar axial charge coupling (time component) can be enhanced by $40-50 \%$ due to the short-range component of the nucleon-nucleon interaction, whereas the isoscalar axial current coupling (space component) is reduced by about $10 \%$ by the exchange current involving the $\pi a_{0}(980)$ exchange mechanism. It seems interesting to examine the consequences of these effective coupling constants for neutrino-nucleus scatterings.

To summarize, we wish to reemphasize that neutrino-nucleus reactions play an important role in the study of the astrophysical neutrinos. The diversity of possible types of reactions presents a great challenge to nuclear physicists. It is hoped that the present review have succeeded in calling our fellow nuclear physicists' attention to some of the major problems facing us in this field.

\section{Acknowledgement}

We wish to express our sincere thanks to Dr. Y. Kohyama for many valuable consultations on the topics discussed in this review article. At various stages of our work on neutrino-nucleus reactions, we benefited greatly from collaborations and/or discussions with Prof. M. Fukugita, Prof. J. N. Bahcall, Prof. W. C. Haxton and Prof. M. Koshiba, to whom we would like to express our gratitude. We are also indebted to Prof. C. Goodman for useful comments on an early version of the manuscript. 


\section{References}

[1] J.N.Bahcall, Neutrino Astrophysics, Cambridge University Press, 1989.

[2] M.Fukugita, Astrophysical Neutrinos and Nuclear Physics, in Lectures given at the 103rd Enrico Fermi Summer School, 1987 (North-Holland, Amsterdam, 1989) p. 331

[3] M.Koshiba, Phys. Rep. 220, 229 (1992).

[4] J.N.Bahcall and R.K.Ulrich, Rev. Mod. Phys. 60, 297 (1988).

[5] R.Davis, Jr., J.Evans, V.Radeka and L.Roger, Phys. Rev. Lett. 201205 (1968).

[6] J.K.Rowley, B.T.Cleveland and R.Davis, Jr., in Solar Neutrinos and Neutrinos Astronomy, Proceedings of the AIP Conference, Lead, South Dakota, 1984, edited by M.L.Cherry, W.A.Fowker and K.Lande, AIP Conf. Proc. No. 126 (AIP, New York, 1985) 1.

[7] R.Davis, Jr., Proceedings of the Seventh Workship on Grand Unification, ICOBANS'86 Toyama, Japan, edited by J. Arafune (World Scientific, Singaporem 1986), p. 237

[8] R.Davis, Jr., et al., in Proceedings of the Thirteenth International Conferences on Neutrino Physics and Astrophysics, Boston, Massachusetts, 1988, edited by J.Schneps et al. (World Scientific, Singapore, 1989), p. 518.

[9] R.Davis, Jr., K.Lande, C.K.Lee, P.Wilderhain, A.Weinberger, T.Daily, B.Cleveland, and J.Ullman, in Proceedings of the XXI International Cosmic Ray Conference, Adelaide, Australia, 1990, edited by R.J.Protheroe (Graphic Services, Northfield, South Australia, 1990).

[10] K.S.Hirata et al., Phys. Rev. Lett. 63, 16 (1989).

[11] K.S.Hirata et al., Phys. Rev. Lett. 65, 1297 (1990).

[12] K.S.Hirata et al., Phys. Rev. D 44, 2241 (1991). 
[13] P.Anselmann et al., Phys. Lett. B 285,376 (1992).

[14] A.I. Abazov et al., Phys. Rev. Lett. 67, 3332 (1991).

[15] V.N.Gavrin et al., in Proceedings of the XXVI International Conference on High Energy Physics, Dallas, Texas, 1992 (unpublished).

[16] J.N.Bahcall and H.A.Bethe, Phys. Rev. D 47, 1298 (1993).

[17] T.K.Kuo and J.Pentalone, Rev. Mod. Phys. 61, 937 (1989).

[18] S.P.Mikheyev and A.Yu.Smirnov, Yad. Fiz. 42, 1441 (1985) [Sov. J. Nucl. Phys. 42, $913(1985)]$

[19] L.Wolfenstein, Phys. Rev. D17, 2369 (1978).

[20] S.S.Gerstein, N. van Hieu and E.A.Eramzhyan, Zh. Eksp. Teor. Fiz. 43,1554 (1962) [Sov. Phys. JETP 16 (1963) 1097].

[21] T.W.Donnelly and R.E.Peccei, Phys. Rep. 50, 1 (1979).

[22] H.H.Chen, Phys. Rev. Lett. 55, 1534 (1985)

[23] G.A.Ardsma et al., Phys. Lett. B 194, 321 (1987).

[24] G.T.Ewan et al., Sudbury Neutrino Observatory Proposal SNO-87- 12, 1987

[25] W.C.Haxton, Phys. Rev. D 36, 2283 (1987).

[26] B.Sadoulet, J.Rich, M.Spiro and D.O.Caldwell, Ap. J. Lett.. 324, L75 (1988).

[27] A.Drukier and L.Stodolsky, Phys. Rev. D 30, 2295 (1984).

[28] B.Cabrera, L.M.Krauss and F.Wilczek, Phys. Rev. Lett. 55, 25 (1985).

[29] C.J.Martoff, Science, 237, 507 (1987). 
[30] R.E.Marshak, Riazuddin and C.P.Ryan, Theory of Weak Interactions in Particle Physics, (Wiley-Intersciences, New York, 1969).

[31] R.J.Blin-Stoyle, Fundamental Interactions and the Nucleus (Northe-Holland, Amsterdam, 1973).

[32] S.Ellis and J.N.Bahcall, Nucl. Phys. A114, 636 (1968)

[33] S. Nozawa, Y. Kohyama, T. Kaneta and K. Kubodera, J. Phys. Soc. Jpn. 55, 2636 (1986)

[34] J. Bahcall, K. Kubodera and S. Nozawa, Phys. Rev. D 38, 1030 (1988)

[35] S. Ying, W. C. Haxton and E. M. Henley, Phys. Rev. D40, 3211 (1989)

[36] N.Tatara, Y.Kohyama and K.Kubodera, Phys. Rev. C 42, 1694 (1990)

[37] M.Doi and K.Kubodera, Phys. Rev. C 45, 1988 (1992).

[38] S.Ying, W.C.Haxton and E.M.Henley, Phys. Rev. C 45, 1982 (1992).

[39] M.Doi and K.Kubodera, unpublished (1992).

[40] Y.Kohyama and K.Kubodera, USC(NT)-Report-92-1, 1992, unpublished.

[41] S.Willis et al, Phys. Rev. Lett. 44, 522 (1980).

[42] A.B.Balantekin and F.Loreti, Phys. Rev. D 45, 1059 (1992).

[43] J.Carlson, D.O.Riska, R.Schiavilla and R.B.Wiringa, Phys. Rev. C 44, 619 (1991).

[44] M.Gari and A.H.Huffman, Astrophys. J. 178, 543 (1972).

[45] F.Dautry, M.Rho and D.O.Riska, Nucl. Phys. A264, 507 (1976).

[46] C.Werntz and J.G.Brennan, Phys. Rev. C 8, 1545 (1973).

[47] P.E.Tegner and C.Bargholtz, Astrophys. J. 272, 311 (1983). 
[48] C.W.Kim and H.Primakoff, Phys. Rev. 140, B566 (1965);

for a recent review, see C.W.Kim and H.Primakoff, in Mesons in Nuclei, vol.1, edited by M.Rho and D.Wilkinson (North-Holland, Amsterdam, 1979), p.67

[49] A.Fujii and Y.Yamaguchi, Prog. Theor. Phys. 31, 107 (1964).

[50] A.Galindo and P.Pascual, Nucl. Phys. B14 (1969) 37.

[51] J. Delorme, "Sur une méthode globale de traitement des courants nucléaires", thesis, Univ. Claude Bernard (Lyon, 1972), unpublished.

[52] R.C.Allen et al., Phys. Rev. Lett., 64, 1871 (1990).

[53] D.A.Krakauer et al., Phys. Rev. C 45, 2450 (1992).

[54] D.D..Koetke et al., Phys. Rev. C 46, 2554 (1992).

[55] S.J.Freedman et al., Phys. Rev. D 47, 811 (1993), and extensive references therein on the E645 experiment at Los Alamos.

[56] G.Drexlin et al. (KARMEN), in Proc. Intern. Workshop (Heidelberg, October 1987), eds. H.V.Klapdor and B.Povh (Springer, Berlin 1988) p. 147.

[57] B. Zeitnitz, Prog. Part. Nucl. Phys. Vol. 13, ed. A. Faessler (Pergamon, Oxford, 1985) p. 445 .

[58] M.Fukugita, Y.Kohyama and K.Kubodera, Phys. Lett. 212B, 139 (1988).

[59] S.L.Mintz and M.Pourkaviani, Phys. Rev. C 40, 2458 (1989);

[60] M.Pourkaviani and S.L.Mintz, J. Phys. G 16, 569 (1990).

[61] S.L.Mintz and M.Purkaviani, Phys. Rev. C 38, 2443 (1988).

[62] J.Bernabéu and P.Pascual, Nucl. Phys. A324, 365 (1979).

[63] F.Ajzenberg-Selove, Nucl. Phys. A433, 1 (1985). 
[64] M.Morita et al., Prog. Theor. Phys. Suppl. 60, 1 (1976).

[65] W.-Y.Hwang and H.Primakoff, Phys. Rev. C 16, 397 (1977).

[66] L.Grenacs, Annu. Rev. Nucl. Part. Sci. 35, 455 (1985).

[67] Y.Masuda, T.Minamisono, Y.Nojiri and K.Sugimoto, Phys. Rev. Lett. 43, 1083 (1979); T.Minamisono et al., J. Phys. Soc. Jpn. Suppl. 55, 86 (1986).

[68] P.Lebrun et al., Phys. Rev. Lett. 40, 302 (1978); H.Brändle et al., Phys. Rev. Lett. 40, 306 (1978); H.Brändle et al., Phys. Rev. Lett. 41, 299 (1978).

[69] S.Nozawa, Y.Kohyama and K.Kubodera, Prog. Theor. Phys. 70, 892 (1983).

[70] B.Chertok et al., Phys. Rev. C 8, 23 (1973).

[71] J.D.Walecka, in Muon Physics, vol. 2, eds.V.W.Hughes and C.S.Wu (Academic Press, New York, 1975), p. 113.

[72] T.W.Donnelly and J.D.Walecka, Ann. Rev. Nucl. Sci. 25, 329 (1975).

[73] T.W.Donnelly, Phys. Lett. 43B, 93 (1973).

[74] T.W.Donnelly, unpublished, as cited in refs. [53, 78

[75] M.Chemtob and M.Rho, Nucl. Phys. A163, 1 (1971).

[76] G. Drexlin et al., Nucl. Instrum. Methods, A289, 490 (1990);

B.Bodmann et al., Nucl. Instrum. Methods, A286, 214 (1990).

[77] B.Bodmann et al. (KARMEN), Phys. Lett. 267B, 321 (1991).

[78] B.Bodmann et al. (KARMEN), Phys. Lett. B280, 198 (1992).

[79] B.Bodmann et al., Contribution to Neutrino '92.

[80] J.S.O'Connell, T.W.Donnelly and J.D.Walecka, Phys. Rev. C 6, 719 (1972). 
[81] H.Überall, B.A.Lamers, J.B.Langworthy and F.J.Kelly, Phys. Rev. C 6, 1911 (1972).

[82] T.W.Donnelly, Proc. of the Los Alamos Neutrino Workshop, Los Alamos Netional Laboratory Report No. LA-9358-C, 1981 (unpublished).

[83] D.S.Armstrong et al., Phys. Rev. C 46, 1094 (1992).

[84] H.W.Fearing and M.S.Welsh, Phys. Rev. C 46, 2077 (1992).

[85] B.H.Brandow, Rev. Mod. Phys. 39, 771 (1967).

[86] P.J.Ellis and E.Osnes, Rev. Mod. Phys. 49, 777 (1977).

[87] D.H.Wilkinson, Phys. Rev. C 7, 930 (1973); Nucl. Phys. A209, 470 (1973).

[88] D.H.Wilkinson, Nucl. Phys. A225, 365 (1974).

[89] B.A.Brown and B.H.Wildenthal, Phys. Rev. C 28, 2397 (1983).

[90] B.A.Brown and B.H.Wildenthal, At. Data Nucl. Data Tables 33, 347 (1985).

[91] W.-T.Chou, E.K.Warburton and B.A.Brown, Phys. Rev. C 47, 163 (1993).

[92] I.S.Towner and F.C.Khanna, Nucl. Phys. A399, 334 (1983).

[93] I.S.Towner, Phys. Rep. 155, 263 (1987), and an extensive list of references therein.

[94] A.Arima, K.Shimizu, W.Bentz and H.Hyuga, Adv. Nucl. Phys. 18, 1 (1987), and an exhaustive list of references therein.

[95] R.S.Raghavan, S.Pakvasa and B.A.Brown, Phys. Rev. Lett. 57, 1801 (1986).

[96] J.Arafune, M.Fukugita, Y.Kohyama and K.Kubodera, Phys. Lett. 217B, 186 (1989).

[97] F.Ajzebgerg-Selove, Nucl. Phys. A449, 1 (1986).

[98] M.Fukugita, Y.Kohyama, K.Kubodera and T.Kuramoto, Phys. Rev. C 41, 1359 (1990). 
[99] S.Cohen and D.Kurath, Nucl. Phys. 73, 1 (1965).

[100] A.G.M. van Hees, A.A.Wolters and P.W.M.Glaudemans, Nucl. Phys. A476, 61 (1988).

[101] A.A.Wolters et al., Phys. Rev. C 42, 2053 (1990); 42, 2062 (1990).

[102] A.A.Wolters, Thesis (University of Utrecht, 1989), unpublished.

[103] R.S.Raghavan and S.Pakvasa, Phys. Rev. D 37, 849 (1988).

[104] A.Acker, S.Pakvasa and R.S.Raghavan, Phys. Lett. B238, 117 (1990).

[105] R.S.Raghavan, in Proceedings of the 25th International Conference on High Energy Physics, Singapore, 1990, edited by K. K. Phua and Y. Yamaguchi (World Scientific, Singapore, 1991)

[106] R.S.Raghavan, A.B.Balantekin, F.Loreti, A.J.Baltz, S.Pakvasa and J.Pantaleone, Phys. Rev. D 44, 3786 (1991).

[107] C.D.Goodman, C.A.Goulding, M.B.Greenfield, J.Rapaport, D.E.Bainum, C.C.Foster, W.G.Love and F.Petrovich, Phys. Rev. Lett. 44, 1755 (1980).

[108] J.W.Watson, W.Pairsuwan, B.D.Anderson, A.R.Baldwin, B.S.Flanders, R.Madey, R.J.McCarthy, B.A.Brown, B.H.Wildenthal and C.C.Foster, Phys. Rev. Lett. 55, 1369 (1985).

[109] T.N.Tadeucci, C.A.Goulding, T.A.Carey, R.C.Byrd, C.D.Goodman, C.Gaarde, J.Larsen, D.Horen, J.Rapaport and E.Sugarbaker, Nucl. Phys. A469, 125 (1987).

[110] N.Austern, Direct Nuclear Reaction Theory (Wiley, New York, 1970) p. 273.

[111] D.E.Bainum, J.Rapaport, C.D.Goodman, D.J.Horen, C.C.Foster, M.B.Greenfield and C.A.Goulding, Phys. Rev. Lett. 44, 1751 (1980). 
[112] K.Ikeda, S.Fujii and J.I.Fujita, Phys. Lett. 3, 271 (1963).

[113] B.D.Anderson et al., Phys. Rev. C, 31, 1161 (1985).

[114] B.D.Anderson et al., Phys. Rev. C, 36, 2195 (1987)

[115] B.D.Anderson et al., Phys. Rev. C, 43, 50 (1991).

[116] B.A.Brown and B.H.Wildenthal, Ann. Rev. Nucl. Part. Sci. 38, 29 (1988).

[117] J.Rapaport et al., Phys. Rev. Lett. 47, 1518 (1981).

[118] D.Krofcheck et al., Phys. Rev. Lett. 55, 1051 (1985).

[119] D. Krofcheck et al., Phys. Lett. B189, 299 (1987).

[120] J.Rapaport et al., Phys. Rev. Lett. 54, 2325 (1985).

[121] D.Krofchek, unpublished (1987).

[122] W.C.Haxton, Phys. Rev. Lett. 60, 768 (1988).

[123] Proceedings of " $G$-T and Neutrino Cross Section Workshop", edited by K.Lande et al. (April 1993, Philadelphia)

[124] E.R.Sugarbaker, in ref. 123.

[125] P.Wildenhain, in ref. [123].

[126] J.Engel, S.Pittel and P.Vogel, Phys. Rev. Lett. 67, 426 (1991).

[127] H.C.Haxton, in ref. [123].

[128] D.H.Wilkinson, Proceedings of Ecole d'Eté de Physique Théorique, Session XXX (Les Houches, 1977), eds. R.Balian, M.Rho and R.Ripka (North-Holland, Amsterdam, 1978), p. 879 .

[129] W.E.Ormand and B.A.Brown, Nucl. Phys. A491, 1 (1989). 
[130] R.G.Sextro, R.A.Gough and J.Cerny, Nucl. Phys. A234, 130 (1974).

[131] B.H.Wildenthal, Prog.Part. Nucl. Phys. vol. 11 ed. D.H.Wilkinson (Pergamon, Oxford, 1984) p. 5.

[132] E.G.Adelberger and W.C.Haxton, Phys. Rev. C 36, 879 (1987).

[133] A.García et al., Phys. Rev. Lett. 26, 3654 (1991).

[134] E.G.Adelberger, A.García, P.V.Magnus and D.P.Wells, Phys. Rev. Lett. 67, 3658 (1991).

[135] J.Rapaport and E.R.Sugarbaker, Phys. Rev. Lett. 69, 2444 (1992).

[136] C.D.Goodman, M.B.Aufderheide, S.D.Bloom and D.A.Resler, Phys. Rev. Lett. 69, $2445(1992)$.

[137] M.B.Aufderheide, S.D.Bloom, D.A.Resler and C.D.Goodman, Phys. Rev. C 46, 2251 (1992).

[138] D.Wells, Bull. Am. Phys. Soc. 37, 1296 (1992).

[139] B.A.Brown, Phys. Rev. Lett. 69, 1034 (1992).

[140] C.Iliadis, J.Höhne, F.Käppeler, J.Meissner, H.P.Trautvetter and M.Wiescher, Phys. Rev. C (in press).

[141] J.N.Bahcall, Phys. Rev. Lett. 61, 2650 (1988).

[142] K.S.Hirata et al., Phys. Rev. Lett. 61, 2653 (1988).

[143] K.S.Hirata et al., Phys. Lett. B205, 416 (1988); B280, 146 (1992).

[144] R.M.Bionta et al., Phys. Rev. D 38, 768 (1988); R.Becker-Szendy et al., Phys. Rev. D 46, 3720 (1992).

[145] T.deForest and J.D.Walecka, Adv. in Phys. 15, 329 (1975). 
[146] J.N.Bahcall and S.C.Frautschi, Phys. Rev. 136, B1547 (1964).

[147] J.S.Bell and C.H.Llewellyn-Smith, Nucl. Phys. B28, 317 (1971).

[148] R.A.Smith and E.J.Moniz, Nucl. Phys. B43, 605 (1972).

[149] E.V.Bugaev, G.S.Bisnovatyi-Kogan, M.A.Rudzsky and Z.F.Seidov, Nucl. Phys. A324, 350 (1979).

[150] J.S.O'Connell, in Proc. of the Los Alamos Neutrino Workshop, Los Alamos, 1981, edited by F.Boehm and G.J.Stephensen, Jr. (Los Alamos Report No. LA-9358-O, 1982).

[151] T.K.Gaisser and J.S.O'Connell, Phys. Rev. D 34, 822 (1986).

[152] T.K.Gaisser, M.Nowakowski and E.A.Paschos, Phys. Rev. D 33, 1233 (1986).

[153] S.L.Adler, S.Nussinov and E.A.Paschos, Phys. Rev. D 9, 2125 (1974).

[154] M.Fukugita, Y.Kohyama, K.Kubodera and T.Kuramoto, Astrophys. J. 337, L59 (1989)

[155] T.Kuramoto, M.Fukugita, Y.Kohyama and K.Kubodera, Nucl. Phys. A512, 711 (1990).

[156] J.B.Langworthy, B.A.Lamers and H.Überall, Nucl. Phys. A280, 351 (1977).

[157] W.C.Haxton and C.Johnson, Phys. Rev. Lett. 65, 1325 (1990).

[158] E.J.Moniz, Phys. Rev. 184, 1154 (1969).

[159] R.R.Whitney, I.Sick, J.R.Ficence, R.D.Kephart and W.P.Trower, Phys. Rev. C 9, $2230(1974)$

[160] E.J.Moniz et al., Phys. Rev. Lett. 26, 445 (1971).

[161] C.R.Chinn, A.Pickelsimer and J.W.Van Orden, Phys. Rev. C 40, 790 (1989). 
[162] S.K.Singh and E.Oset, preprint (Univeristy of Valencia), to appear in Phys. Rev. C

[163] A.K.Mann, Phys. Rev. D 48, 422 (1993).

[164] Table of Isotopes (7th edition), ed. C.M.Lederer and V.S.Shirley (Wiley, New York, 1978)

[165] T.W.Donnelly and W.C.Haxton, Nucl. Phys. A287, 506 (1977).

[166] J.N.Bahcall and W.H.Press, Astrophys. J. 370, 730 (1991).

[167] L.M.Krauss, Nature 329, 689 (1987).

[168] J.W.Bieber, D.Steckel, T.Stanev and G.Steigmann, Nature 348, 408 (1990).

[169] J.N.Bahcall, G.B.Field and W.H.Press, Astrophys. J. 320, L69 (1987).

[170] G.A.Koval'tsov, Izv. Akad. Nauk SSSR, Ser. Fiz. 45, 1115 (1981) (Bull. Acad. Sci. USSR, Phys. Ser. 45, 23 (1981)).

[171] L.A.Ahrens et al., Phys. Lett. B202, 284 (1988).

[172] M.Oka and K.Kubodera, Phys. Lett., 90B, 45 (1980).

[173] J. Ashman et al. (the EMC collaboration), Phys. Lett. B206, 364 (1988); Nucl. Phys. B328, 1 (1989)

[174] M.Bourquin et al., Z. Phys. C21, 27 (1983).

[175] J.F. Donoghue and C.R. Nappi, Phys. Lett. B168, 105 (1986).

[176] J. Gasser, H. Leutwyler and M.E. Sainio, Phys. Lett. B253, 252 (1991).

[177] E.J.Beise and R.D.McKeown, Comm. on Nucl. Part. Phys. 20, 105 (1991).

[178] L.A.Ahrens et al., Phys. Rev. D 35, 785 (1987).

[179] D.Kaplan and A.Manohar, Nucl. Phys. B310, 527 (1988). 
[180] E.M.Henley, G.Krein, S.J.Pollock and A.G.Williams, Phys. Lett. B269, 31 (1991).

[181] T.Suzuki, Y.Kohyama and K.Yazaki, Phys. Lett. B252, 323 (1990).

[182] J.Bernabéu, T.E.O.Ericson, E.Hernández and J.Ros, Nucl. Phys. B378, 131 (1992).

[183] M.Hjorth-Jensen, M.Kirchbach, R.O.Riska and K.Tsushima, Nucl. Phys. A (in press). 
Table 1 Neutrino-deuteron cross sections in units of $\mathrm{cm}^{2}$

\begin{tabular}{|c|c|c|c|c|}
\hline$E_{\nu}(\mathrm{MeV})$ & $\nu d \rightarrow \nu^{\prime} p n$ & $\bar{\nu} d \rightarrow \bar{\nu}^{\prime} p n$ & $\nu d \rightarrow e^{-} p p$ & $\bar{\nu} d \rightarrow e^{+} n n$ \\
\hline 2.0 & $0.000 \mathrm{E}+00$ & $0.000 \mathrm{E}+00$ & $3.854 \mathrm{E}-45$ & $0.000 \mathrm{E}+00$ \\
2.2 & $0.000 \mathrm{E}+00$ & $0.000 \mathrm{E}+00$ & $8.231 \mathrm{E}-45$ & $0.000 \mathrm{E}+00$ \\
2.4 & $4.394 \mathrm{E}-47$ & $4.362 \mathrm{E}-47$ & $1.461 \mathrm{E}-44$ & $0.000 \mathrm{E}+00$ \\
2.6 & $4.288 \mathrm{E}-46$ & $4.253 \mathrm{E}-46$ & $2.318 \mathrm{E}-44$ & $0.000 \mathrm{E}+00$ \\
2.8 & $1.464 \mathrm{E}-45$ & $1.451 \mathrm{E}-45$ & $3.413 \mathrm{E}-44$ & $0.000 \mathrm{E}+00$ \\
3.0 & $3.369 \mathrm{E}-45$ & $3.334 \mathrm{E}-45$ & $4.761 \mathrm{E}-44$ & $0.000 \mathrm{E}+00$ \\
3.2 & $6.308 \mathrm{E}-45$ & $6.236 \mathrm{E}-45$ & $6.377 \mathrm{E}-44$ & $0.000 \mathrm{E}+00$ \\
3.4 & $1.041 \mathrm{E}-44$ & $1.028 \mathrm{E}-44$ & $8.274 \mathrm{E}-44$ & $0.000 \mathrm{E}+00$ \\
3.6 & $1.579 \mathrm{E}-44$ & $1.557 \mathrm{E}-44$ & $1.046 \mathrm{E}-43$ & $0.000 \mathrm{E}+00$ \\
3.8 & $2.252 \mathrm{E}-44$ & $2.219 \mathrm{E}-44$ & $1.296 \mathrm{E}-43$ & $0.000 \mathrm{E}+00$ \\
4.0 & $3.068 \mathrm{E}-44$ & $3.021 \mathrm{E}-44$ & $1.577 \mathrm{E}-43$ & $0.000 \mathrm{E}+00$ \\
4.2 & $4.034 \mathrm{E}-44$ & $3.967 \mathrm{E}-44$ & $1.890 \mathrm{E}-43$ & $1.065 \mathrm{E}-45$ \\
4.4 & $5.154 \mathrm{E}-44$ & $5.064 \mathrm{E}-44$ & $2.237 \mathrm{E}-43$ & $4.397 \mathrm{E}-45$ \\
4.6 & $6.434 \mathrm{E}-44$ & $6.314 \mathrm{E}-44$ & $2.618 \mathrm{E}-43$ & $9.832 \mathrm{E}-45$ \\
4.8 & $7.877 \mathrm{E}-44$ & $7.722 \mathrm{E}-44$ & $3.033 \mathrm{E}-43$ & $1.748 \mathrm{E}-44$ \\
5.0 & $9.487 \mathrm{E}-44$ & $9.290 \mathrm{E}-44$ & $3.485 \mathrm{E}-43$ & $2.747 \mathrm{E}-44$ \\
5.2 & $1.127 \mathrm{E}-43$ & $1.102 \mathrm{E}-43$ & $3.973 \mathrm{E}-43$ & $3.993 \mathrm{E}-44$ \\
5.4 & $1.322 \mathrm{E}-43$ & $1.292 \mathrm{E}-43$ & $4.498 \mathrm{E}-43$ & $5.498 \mathrm{E}-44$ \\
5.6 & $1.534 \mathrm{E}-43$ & $1.498 \mathrm{E}-43$ & $5.060 \mathrm{E}-43$ & $7.270 \mathrm{E}-44$ \\
5.8 & $1.765 \mathrm{E}-43$ & $1.721 \mathrm{E}-43$ & $5.661 \mathrm{E}-43$ & $9.319 \mathrm{E}-44$ \\
6.0 & $2.013 \mathrm{E}-43$ & $1.961 \mathrm{E}-43$ & $6.239 \mathrm{E}-43$ & $1.165 \mathrm{E}-43$ \\
6.2 & $2.279 \mathrm{E}-43$ & $2.218 \mathrm{E}-43$ & $6.904 \mathrm{E}-43$ & $1.427 \mathrm{E}-43$ \\
6.4 & $2.563 \mathrm{E}-43$ & $2.491 \mathrm{E}-43$ & $7.606 \mathrm{E}-43$ & $1.719 \mathrm{E}-43$ \\
6.6 & $2.866 \mathrm{E}-43$ & $2.782 \mathrm{E}-43$ & $8.347 \mathrm{E}-43$ & $2.040 \mathrm{E}-43$ \\
6.8 & $3.198 \mathrm{E}-43$ & $3.102 \mathrm{E}-43$ & $9.127 \mathrm{E}-43$ & $2.392 \mathrm{E}-43$ \\
7.0 & $3.541 \mathrm{E}-43$ & $3.430 \mathrm{E}-43$ & $9.945 \mathrm{E}-43$ & $2.774 \mathrm{E}-43$ \\
7.2 & $3.902 \mathrm{E}-43$ & $3.776 \mathrm{E}-43$ & $1.080 \mathrm{E}-42$ & $3.187 \mathrm{E}-43$ \\
7.4 & $4.283 \mathrm{E}-43$ & $4.140 \mathrm{E}-43$ & $1.170 \mathrm{E}-42$ & $3.631 \mathrm{E}-43$ \\
7.6 & $4.683 \mathrm{E}-43$ & $4.522 \mathrm{E}-43$ & $1.264 \mathrm{E}-42$ & $4.106 \mathrm{E}-43$ \\
7.8 & $5.103 \mathrm{E}-43$ & $4.921 \mathrm{E}-43$ & $1.361 \mathrm{E}-42$ & $4.612 \mathrm{E}-43$ \\
\hline
\end{tabular}


Table 1 (continued) Neutrino-deuteron cross sections in units of $\mathrm{cm}^{2}$

\begin{tabular}{|c|c|c|c|c|}
\hline$E_{\nu}(\mathrm{MeV})$ & $\nu d \rightarrow \nu^{\prime} p n$ & $\bar{\nu} d \rightarrow \bar{\nu}^{\prime} p n$ & $\nu d \rightarrow e^{-} p p$ & $\bar{\nu} d \rightarrow e^{+} n n$ \\
\hline 8.0 & $5.542 \mathrm{E}-43$ & $5.339 \mathrm{E}-43$ & $1.463 \mathrm{E}-42$ & $5.150 \mathrm{E}-43$ \\
\hline 8.2 & $6.000 \mathrm{E}-43$ & $5.775 \mathrm{E}-43$ & $1.569 \mathrm{E}-42$ & $5.719 \mathrm{E}-43$ \\
\hline 8.4 & $6.479 \mathrm{E}-43$ & $6.228 \mathrm{E}-43$ & $1.679 \mathrm{E}-42$ & $6.320 \mathrm{E}-43$ \\
\hline 8.6 & $6.977 \mathrm{E}-43$ & $6.700 \mathrm{E}-43$ & $1.793 \mathrm{E}-42$ & $6.967 \mathrm{E}-43$ \\
\hline 8.8 & $7.495 \mathrm{E}-43$ & $7.189 \mathrm{E}-43$ & $1.911 \mathrm{E}-42$ & $7.635 \mathrm{E}-43$ \\
\hline 9.0 & $8.033 \mathrm{E}-43$ & $7.697 \mathrm{E}-43$ & $2.033 \mathrm{E}-42$ & $8.336 \mathrm{E}-43$ \\
\hline 9.2 & $8.591 \mathrm{E}-43$ & $8.223 \mathrm{E}-43$ & $2.160 \mathrm{E}-42$ & $9.068 \mathrm{E}-43$ \\
\hline 9.4 & $9.169 \mathrm{E}-43$ & 8.767E-43 & $2.290 \mathrm{E}-42$ & $9.833 \mathrm{E}-43$ \\
\hline 9.6 & $9.767 \mathrm{E}-43$ & $9.328 \mathrm{E}-43$ & $2.425 \mathrm{E}-42$ & $1.063 \mathrm{E}-42$ \\
\hline 9.8 & $1.039 \mathrm{E}-42$ & $9.908 \mathrm{E}-43$ & $2.564 \mathrm{E}-42$ & $1.146 \mathrm{E}-42$ \\
\hline 10.0 & $1.103 \mathrm{E}-42$ & $1.051 \mathrm{E}-42$ & $2.708 \mathrm{E}-42$ & $1.232 \mathrm{E}-42$ \\
\hline 10.2 & $1.168 \mathrm{E}-42$ & $1.112 \mathrm{E}-42$ & $2.855 \mathrm{E}-42$ & $1.322 \mathrm{E}-42$ \\
\hline 10.4 & $1.236 \mathrm{E}-42$ & $1.176 \mathrm{E}-42$ & $3.007 \mathrm{E}-42$ & $1.415 \mathrm{E}-42$ \\
\hline 10.6 & $1.306 \mathrm{E}-42$ & $1.241 \mathrm{E}-42$ & $3.163 \mathrm{E}-42$ & $1.511 \mathrm{E}-42$ \\
\hline 10.8 & $1.378 \mathrm{E}-42$ & $1.308 \mathrm{E}-42$ & $3.323 \mathrm{E}-42$ & $1.610 \mathrm{E}-42$ \\
\hline 11.0 & $1.453 \mathrm{E}-42$ & $1.377 \mathrm{E}-42$ & $3.488 \mathrm{E}-42$ & $1.712 \mathrm{E}-42$ \\
\hline 11.2 & $1.529 \mathrm{E}-42$ & $1.447 \mathrm{E}-42$ & $3.657 \mathrm{E}-42$ & $1.818 \mathrm{E}-42$ \\
\hline 11.4 & $1.607 \mathrm{E}-42$ & $1.520 \mathrm{E}-42$ & $3.831 \mathrm{E}-42$ & $1.927 \mathrm{E}-42$ \\
\hline 11.6 & $1.688 \mathrm{E}-42$ & $1.594 \mathrm{E}-42$ & $4.008 \mathrm{E}-42$ & $2.039 \mathrm{E}-42$ \\
\hline 11.8 & $1.770 \mathrm{E}-42$ & $1.670 \mathrm{E}-42$ & $4.191 \mathrm{E}-42$ & $2.155 \mathrm{E}-42$ \\
\hline 12.0 & $1.854 \mathrm{E}-42$ & $1.748 \mathrm{E}-42$ & $4.377 \mathrm{E}-42$ & $2.273 \mathrm{E}-42$ \\
\hline 12.5 & $2.075 \mathrm{E}-42$ & $1.950 \mathrm{E}-42$ & $4.863 \mathrm{E}-42$ & $2.584 \mathrm{E}-42$ \\
\hline 13.0 & $2.308 \mathrm{E}-42$ & $2.164 \mathrm{E}-42$ & $5.378 \mathrm{E}-42$ & $2.914 \mathrm{E}-42$ \\
\hline 13.5 & $2.555 \mathrm{E}-42$ & $2.389 \mathrm{E}-42$ & $5.920 \mathrm{E}-42$ & $3.265 \mathrm{E}-42$ \\
\hline 14.0 & $2.815 \mathrm{E}-42$ & $2.625 \mathrm{E}-42$ & $6.491 \mathrm{E}-42$ & $3.635 \mathrm{E}-42$ \\
\hline 14.5 & $3.088 \mathrm{E}-42$ & $2.872 \mathrm{E}-42$ & $7.090 \mathrm{E}-42$ & $4.025 \mathrm{E}-42$ \\
\hline 15.0 & $3.375 \mathrm{E}-42$ & $3.130 \mathrm{E}-42$ & $7.719 \mathrm{E}-42$ & $4.435 \mathrm{E}-42$ \\
\hline 15.5 & $3.675 \mathrm{E}-42$ & $3.399 \mathrm{E}-42$ & $8.376 \mathrm{E}-42$ & $4.864 \mathrm{E}-42$ \\
\hline 16.0 & $3.989 \mathrm{E}-42$ & $3.679 \mathrm{E}-42$ & $9.063 \mathrm{E}-42$ & $5.313 \mathrm{E}-42$ \\
\hline 16.5 & $4.316 \mathrm{E}-42$ & $3.969 \mathrm{E}-42$ & $9.780 \mathrm{E}-42$ & $5.780 \mathrm{E}-42$ \\
\hline 17.0 & $4.656 \mathrm{E}-42$ & $4.271 \mathrm{E}-42$ & $1.053 \mathrm{E}-41$ & $6.267 \mathrm{E}-42$ \\
\hline 17.5 & $5.010 \mathrm{E}-42$ & $4.584 \mathrm{E}-42$ & $1.130 \mathrm{E}-41$ & $6.773 \mathrm{E}-42$ \\
\hline 18.0 & $5.378 \mathrm{E}-42$ & $4.907 \mathrm{E}-42$ & $1.211 \mathrm{E}-41$ & 7.297E-42 \\
\hline 18.5 & $5.760 \mathrm{E}-42$ & $5.241 \mathrm{E}-42$ & $1.295 \mathrm{E}-41$ & $7.841 \mathrm{E}-42$ \\
\hline 19.0 & $6.155 \mathrm{E}-42$ & $5.585 \mathrm{E}-42$ & $1.381 \mathrm{E}-41$ & $8.402 \mathrm{E}-42$ \\
\hline 19.5 & $6.564 \mathrm{E}-42$ & $5.940 \mathrm{E}-42$ & $1.471 \mathrm{E}-41$ & $8.983 \mathrm{E}-42$ \\
\hline
\end{tabular}


Table 1 (continued) Neutrino-deuteron cross sections in units of $\mathrm{cm}^{2}$

\begin{tabular}{|c|c|c|c|c|}
\hline$E_{\nu}(\mathrm{MeV})$ & $\nu d \rightarrow \nu^{\prime} p n$ & $\bar{\nu} d \rightarrow \bar{\nu}^{\prime} p n$ & $\nu d \rightarrow e^{-} p p$ & $\bar{\nu} d \rightarrow e^{+} n n$ \\
\hline 20.0 & $6.987 \mathrm{E}-42$ & $6.306 \mathrm{E}-42$ & $1.564 \mathrm{E}-41$ & $9.581 \mathrm{E}-42$ \\
20.5 & $7.424 \mathrm{E}-42$ & $6.682 \mathrm{E}-42$ & $1.661 \mathrm{E}-41$ & $1.020 \mathrm{E}-41$ \\
21.0 & $7.875 \mathrm{E}-42$ & $7.069 \mathrm{E}-42$ & $1.760 \mathrm{E}-41$ & $1.083 \mathrm{E}-41$ \\
21.5 & $8.339 \mathrm{E}-42$ & $7.466 \mathrm{E}-42$ & $1.862 \mathrm{E}-41$ & $1.149 \mathrm{E}-41$ \\
22.0 & $8.818 \mathrm{E}-42$ & $7.873 \mathrm{E}-42$ & $1.968 \mathrm{E}-41$ & $1.216 \mathrm{E}-41$ \\
22.5 & $9.311 \mathrm{E}-42$ & $8.291 \mathrm{E}-42$ & $2.077 \mathrm{E}-41$ & $1.285 \mathrm{E}-41$ \\
23.0 & $9.818 \mathrm{E}-42$ & $8.719 \mathrm{E}-42$ & $2.189 \mathrm{E}-41$ & $1.355 \mathrm{E}-41$ \\
23.5 & $1.034 \mathrm{E}-41$ & $9.157 \mathrm{E}-42$ & $2.305 \mathrm{E}-41$ & $1.427 \mathrm{E}-41$ \\
24.0 & $1.087 \mathrm{E}-41$ & $9.606 \mathrm{E}-42$ & $2.423 \mathrm{E}-41$ & $1.501 \mathrm{E}-41$ \\
24.5 & $1.142 \mathrm{E}-41$ & $1.006 \mathrm{E}-41$ & $2.545 \mathrm{E}-41$ & $1.577 \mathrm{E}-41$ \\
25 & $1.199 \mathrm{E}-41$ & $1.053 \mathrm{E}-41$ & $2.671 \mathrm{E}-41$ & $1.655 \mathrm{E}-41$ \\
26 & $1.316 \mathrm{E}-41$ & $1.150 \mathrm{E}-41$ & $2.932 \mathrm{E}-41$ & $1.815 \mathrm{E}-41$ \\
27 & $1.439 \mathrm{E}-41$ & $1.251 \mathrm{E}-41$ & $3.206 \mathrm{E}-41$ & $1.981 \mathrm{E}-41$ \\
28 & $1.567 \mathrm{E}-41$ & $1.355 \mathrm{E}-41$ & $3.494 \mathrm{E}-41$ & $2.155 \mathrm{E}-41$ \\
29 & $1.702 \mathrm{E}-41$ & $1.464 \mathrm{E}-41$ & $3.796 \mathrm{E}-41$ & $2.335 \mathrm{E}-41$ \\
30 & $1.842 \mathrm{E}-41$ & $1.576 \mathrm{E}-41$ & $4.112 \mathrm{E}-41$ & $2.521 \mathrm{E}-41$ \\
31 & $1.988 \mathrm{E}-41$ & $1.692 \mathrm{E}-41$ & $4.443 \mathrm{E}-41$ & $2.713 \mathrm{E}-41$ \\
32 & $2.140 \mathrm{E}-41$ & $1.812 \mathrm{E}-41$ & $4.787 \mathrm{E}-41$ & $2.912 \mathrm{E}-41$ \\
33 & $2.298 \mathrm{E}-41$ & $1.936 \mathrm{E}-41$ & $5.146 \mathrm{E}-41$ & $3.117 \mathrm{E}-41$ \\
34 & $2.462 \mathrm{E}-41$ & $2.063 \mathrm{E}-41$ & $5.520 \mathrm{E}-41$ & $3.328 \mathrm{E}-41$ \\
35 & $2.632 \mathrm{E}-41$ & $2.194 \mathrm{E}-41$ & $5.909 \mathrm{E}-41$ & $3.545 \mathrm{E}-41$ \\
36 & $2.808 \mathrm{E}-41$ & $2.329 \mathrm{E}-41$ & $6.313 \mathrm{E}-41$ & $3.768 \mathrm{E}-41$ \\
37 & $2.990 \mathrm{E}-41$ & $2.467 \mathrm{E}-41$ & $6.731 \mathrm{E}-41$ & $3.996 \mathrm{E}-41$ \\
38 & $3.178 \mathrm{E}-41$ & $2.609 \mathrm{E}-41$ & $7.165 \mathrm{E}-41$ & $4.231 \mathrm{E}-41$ \\
39 & $3.372 \mathrm{E}-41$ & $2.754 \mathrm{E}-41$ & $7.614 \mathrm{E}-41$ & $4.470 \mathrm{E}-41$ \\
40 & $3.572 \mathrm{E}-41$ & $2.902 \mathrm{E}-41$ & $8.079 \mathrm{E}-41$ & $4.716 \mathrm{E}-41$ \\
41 & $3.778 \mathrm{E}-41$ & $3.055 \mathrm{E}-41$ & $8.559 \mathrm{E}-41$ & $4.966 \mathrm{E}-41$ \\
42 & $3.991 \mathrm{E}-41$ & $3.210 \mathrm{E}-41$ & $9.055 \mathrm{E}-41$ & $5.222 \mathrm{E}-41$ \\
43 & $4.209 \mathrm{E}-41$ & $3.368 \mathrm{E}-41$ & $9.567 \mathrm{E}-41$ & $5.484 \mathrm{E}-41$ \\
44 & $4.434 \mathrm{E}-41$ & $3.530 \mathrm{E}-41$ & $1.009 \mathrm{E}-40$ & $5.750 \mathrm{E}-41$ \\
45 & $4.665 \mathrm{E}-41$ & $3.695 \mathrm{E}-41$ & $1.064 \mathrm{E}-40$ & $6.021 \mathrm{E}-41$ \\
46 & $4.901 \mathrm{E}-41$ & $3.864 \mathrm{E}-41$ & $1.120 \mathrm{E}-40$ & $6.298 \mathrm{E}-41$ \\
47 & $5.144 \mathrm{E}-41$ & $4.035 \mathrm{E}-41$ & $1.177 \mathrm{E}-40$ & $6.579 \mathrm{E}-41$ \\
48 & $5.393 \mathrm{E}-41$ & $4.210 \mathrm{E}-41$ & $1.237 \mathrm{E}-40$ & $6.865 \mathrm{E}-41$ \\
49 & $5.649 \mathrm{E}-41$ & $4.387 \mathrm{E}-41$ & $1.297 \mathrm{E}-40$ & $7.156 \mathrm{E}-41$ \\
\hline & & & &
\end{tabular}


Table 1 (continued) Neutrino-deuteron cross sections in units of $\mathrm{cm}^{2}$

\begin{tabular}{|c|c|c|c|c|}
\hline$E_{\nu}(\mathrm{MeV})$ & $\nu d \rightarrow \nu^{\prime} p n$ & $\bar{\nu} d \rightarrow \bar{\nu}^{\prime} p n$ & $\nu d \rightarrow e^{-} p p$ & $\bar{\nu} d \rightarrow e^{+} n n$ \\
\hline 50 & $5.910 \mathrm{E}-41$ & $4.568 \mathrm{E}-41$ & $1.360 \mathrm{E}-40$ & $7.451 \mathrm{E}-41$ \\
51 & $6.177 \mathrm{E}-41$ & $4.751 \mathrm{E}-41$ & $1.424 \mathrm{E}-40$ & $7.751 \mathrm{E}-41$ \\
52 & $6.451 \mathrm{E}-41$ & $4.937 \mathrm{E}-41$ & $1.490 \mathrm{E}-40$ & $8.055 \mathrm{E}-41$ \\
53 & $6.731 \mathrm{E}-41$ & $5.126 \mathrm{E}-41$ & $1.557 \mathrm{E}-40$ & $8.364 \mathrm{E}-41$ \\
54 & $7.017 \mathrm{E}-41$ & $5.318 \mathrm{E}-41$ & $1.626 \mathrm{E}-40$ & $8.676 \mathrm{E}-41$ \\
55 & $7.309 \mathrm{E}-41$ & $5.513 \mathrm{E}-41$ & $1.697 \mathrm{E}-40$ & $8.993 \mathrm{E}-41$ \\
60 & $8.860 \mathrm{E}-41$ & $6.525 \mathrm{E}-41$ & $2.076 \mathrm{E}-40$ & $1.064 \mathrm{E}-40$ \\
65 & $1.056 \mathrm{E}-40$ & $7.599 \mathrm{E}-41$ & $2.498 \mathrm{E}-40$ & $1.237 \mathrm{E}-40$ \\
70 & $1.241 \mathrm{E}-40$ & $8.728 \mathrm{E}-41$ & $2.961 \mathrm{E}-40$ & $1.419 \mathrm{E}-40$ \\
75 & $1.440 \mathrm{E}-40$ & $9.906 \mathrm{E}-41$ & $3.467 \mathrm{E}-40$ & $1.607 \mathrm{E}-40$ \\
80 & $1.654 \mathrm{E}-40$ & $1.113 \mathrm{E}-40$ & $4.014 \mathrm{E}-40$ & $1.801 \mathrm{E}-40$ \\
85 & $1.881 \mathrm{E}-40$ & $1.239 \mathrm{E}-40$ & $4.603 \mathrm{E}-40$ & $2.001 \mathrm{E}-40$ \\
90 & $2.121 \mathrm{E}-40$ & $1.368 \mathrm{E}-40$ & $5.232 \mathrm{E}-40$ & $2.205 \mathrm{E}-40$ \\
95 & $2.373 \mathrm{E}-40$ & $1.500 \mathrm{E}-40$ & $5.900 \mathrm{E}-40$ & $2.412 \mathrm{E}-40$ \\
100 & $2.637 \mathrm{E}-40$ & $1.634 \mathrm{E}-40$ & $6.606 \mathrm{E}-40$ & $2.623 \mathrm{E}-40$ \\
110 & $3.199 \mathrm{E}-40$ & $1.908 \mathrm{E}-40$ & $8.127 \mathrm{E}-40$ & $3.049 \mathrm{E}-40$ \\
120 & $3.801 \mathrm{E}-40$ & $2.186 \mathrm{E}-40$ & $9.784 \mathrm{E}-40$ & $3.481 \mathrm{E}-40$ \\
130 & $4.437 \mathrm{E}-40$ & $2.465 \mathrm{E}-40$ & $1.156 \mathrm{E}-39$ & $3.914 \mathrm{E}-40$ \\
140 & $5.103 \mathrm{E}-40$ & $2.744 \mathrm{E}-40$ & $1.345 \mathrm{E}-39$ & $4.346 \mathrm{E}-40$ \\
150 & $5.793 \mathrm{E}-40$ & $3.020 \mathrm{E}-40$ & $1.543 \mathrm{E}-39$ & $4.776 \mathrm{E}-40$ \\
160 & $6.501 \mathrm{E}-40$ & $3.292 \mathrm{E}-40$ & $1.750 \mathrm{E}-39$ & $5.201 \mathrm{E}-40$ \\
170 & $7.224 \mathrm{E}-40$ & $3.559 \mathrm{E}-40$ & $1.963 \mathrm{E}-39$ & $5.623 \mathrm{E}-40$ \\
\hline
\end{tabular}

